\title{
Nonzero-Sum Stochastic Differential Portfolio Games under a Markovian Regime Switching Model
}

\author{
Chaoqun Ma, ${ }^{1}$ Hui Wu, ${ }^{1}$ and Xiang Lin $^{2}$ \\ ${ }^{1}$ Business School of Hunan University, Changsha 410082, China \\ ${ }^{2}$ School of Finance, Zhejiang Gongshang University, Hangzhou 310018, China \\ Correspondence should be addressed to Hui Wu; h_wu1018@163.com
}

Received 27 September 2014; Accepted 15 December 2014

Academic Editor: Chuangxia Huang

Copyright (c) 2015 Chaoqun Ma et al. This is an open access article distributed under the Creative Commons Attribution License, which permits unrestricted use, distribution, and reproduction in any medium, provided the original work is properly cited.

\begin{abstract}
We consider a nonzero-sum stochastic differential portfolio game problem in a continuous-time Markov regime switching environment when the price dynamics of the risky assets are governed by a Markov-modulated geometric Brownian motion $(\mathrm{GBM})$. The market parameters, including the bank interest rate and the appreciation and volatility rates of the risky assets, switch over time according to a continuous-time Markov chain. We formulate the nonzero-sum stochastic differential portfolio game problem as two utility maximization problems of the sum process between two investors' terminal wealth. We derive a pair of regime switching Hamilton-Jacobi-Bellman (HJB) equations and two systems of coupled HJB equations at different regimes. We obtain explicit optimal portfolio strategies and Feynman-Kac representations of the two value functions. Furthermore, we solve the system of coupled HJB equations explicitly in a special case where there are only two states in the Markov chain. Finally we provide comparative statics and numerical simulation analysis of optimal portfolio strategies and investigate the impact of regime switching on optimal portfolio strategies.
\end{abstract}

\section{Introduction}

The optimal portfolio selection has been studied extensively in modern finance. This research is of great importance from both theoretical and practical purposes. The pioneering work can be traced to Markowitz [1], which provided quantitative methods to investigate a single-period optimal portfolio selection problem. Then Merton [2,3] first extended this single-period model to a continuous-time model. In the seminal work of Merton, he obtained closed-form solutions to the optimal portfolio selection problems. Merton's work has opened up an important field called continuous-time finance. We note that one of the key assumptions in Merton's optimal portfolio models is that the model parameters are assumed to be constants and the price processes of risky assets are modeled by the classical geometric Brownian motions. However, we know that this key assumption is not consistent with the actual behavior of asset price dynamics. Empirical finance literature has found lots of stylized facts in asset returns, such as heavy tails in the asset returns' distributions, time-varying volatility, long-term memory, and regime switching. Thus it would be of practical relevance and importance to consider more realistic portfolio selection models.

In the past three decades or so, among those established Merton's portfolio models, Markov regime switching and differential game models are two main extensions. Regime switching models are an efficient and convenient approach to capture the cyclical features of structure changes in real macroeconomic fundamentals. Early works on regime switching models can be traced to Quandt [4]. Hamilton [5] pioneered the econometric applications of Markov regime switching models. Since then, there has been a growing interest in applications of regime switching models into finance and economics. Guo et al. [6] built and solved a real option model of investment decisions in which the growth rate and volatility of decision variable such as growth rate and diffusion coefficient shift between different states at 
random times. References [7-9] established a dynamic capital structure model and demonstrated how business-cycle variations (regime switching) in expected growth rates, economic uncertainty, and risk premia influence firms' financing and default policies. Some papers on optimal portfolio selection under regime switching models can refer to [10-16] and others. However, it seems that one important issue that may be overlooked by the existing literature on portfolio selection is interactive decision making problems. In this paper we develop a model of two investors to study interactive decision making on portfolio selection.

The origin of the differential game theory might trace to the 1940s. In 1965, Isaacs wrote the classical work "Differential Game." It has laid down the solid mathematical and theoretical foundations to the differential game theory. Since then, stochastic differential game models have found a wide range of applications in finance. Some early works include [1719] and some references therein. Some recent works include [20-24]. Browne [20] formulated various versions of a zerosum stochastic differential game to investigate dynamic optimal investment problems between two "small" investors in continuous time. He provided the existence conditions of Nash equilibrium and gave explicit representations for the value functions and optimal portfolio strategies. Mannucci [25] studied Nash equilibrium for two-player, nonzero-sum stochastic differential game. Elliott and Siu [26] extended the model to a continuous-time Markovian regime switching setting and continued to study the risk minimization portfolio selection problem by using stochastic differential game. Siu [27] considered option pricing under regime switching by a game theoretical approach. More recently, Leong and Huang [28] developed a stochastic differential game of capitalism to study the role of uncertainty. Lin [29] studied a nonzero-sum stochastic differential portfolio game between two investors. Elliott and Siu [30] introduced a model which covered economic risk, financial risk, insurance risk, and model risk to discuss an optimal investment problem of an insurance company using stochastic differential game approach. Liu and Yiu [31] considered stochastic differential games with VaR risk constraints between two insurance companies. They provided explicit Nash equilibria and derived closed-form solutions to value functions. However, It seems that the literature has not well studied the optimal portfolio interactive decision making problem under stochastic differential game in a continuous-time Markovian regime switching setting.

To the best of our knowledge, our model is most related with Browne [20] and Lin [29], since the two papers have discussed stochastic differential portfolio games. However, the distinction between them and our paper is also evident. There are two fundamental differences between the model considered by them and the models considered here. Firstly, we incorporate our model with a general continuous-time Markov regime switching setting and take into account the Markov regime switching risk and its impacts on the financial asset prices. Secondly, we consider a nonzero-sum stochastic differential portfolio game which is different from the several versions of zero-sum stochastic differential game in Browne [20]. Furthermore, we use a stochastic optimal control approach for the current nonzero-sum stochastic differential portfolio game problem. This method is different from the approach used in Lin [29], namely, stochastic linear quadratic control.

In this paper we treat the optimal portfolio selection problem in a wide class of continuous-time Markovian regime switching models. We consider the portfolio selection between two "small" investors; call them A and B. (The investors are called "small" as their decision making behavior does not affect the market prices of the underlying assets.) We consider a continuous-time financial market with three primitive securities, namely, a bank account and two risky assets. The dynamic price processes of all the primitive securities are assumed to be modulated by a continuoustime Markovian chain. The rationale of using this regime switching model is to incorporate the impact of regime shifts on asset prices attributed to structure changes in market or macroeconomy. The two risky assets are correlated with each other, only one of which is available to each investor. Moreover, both investors are allowed to trade freely in the bank account. The investors cooperate with each other by the choice of their own portfolio strategies when they make decisions on investment. We formulate the stochastic differential game as two utility maximization problems. Two objective functions are considered here. One investor is trying to maximize his payoff; simultaneously the other investor acts antagonistically to maximize the other payoff. Each payoff is formulated as expected utility of the wealth sum process of the two investors. By using stochastic optimal control theory, we derive a pair of regime switching HJB equations for the value functions. Moreover, we obtain the Feynman-Kac representations of value functions. Closed-form expressions for optimal portfolio strategies are also obtained. Finally, we find that Markov regime switching in the model parameters has a significant effect on the optimal portfolio strategies and value functions.

Aside from the intrinsic probabilistic and game theoretic interest, such a model is applicable in many economic settings. As we know, diversification improves the ability of an investor's risk-return trade-off. However, it can be difficult for a small investor to hold enough stocks which are well diversified. In addition, maintaining a well-diversified portfolio can lead to high transaction costs. If several investors form a group, well-diversified portfolio and low transaction costs can be realized. Different investors have different attitudes towards risk, so the choice of the stock and the goal of investment for investors are different with each other.

The rest of the paper is organized as follows. The following section presents the price and wealth dynamic processes in a continuous-time Markov regime switching economy. In Section 3, we first introduce two optimal portfolio problems with different objective functions. And then we formulate a two-investor, nonzero-sum stochastic differential portfolio game problem. In Section 4, we derive a pair of regime switching Hamilton-Jacobi-Bellman (HJB) equations for the nonzero-sum differential game problem and explicit solutions for the optimal portfolio strategies and value functions of two investors are obtained. In Section 5 we discuss one special case for a two-state Markov regime switching model. In Section 6 we provide the comparative statics and numerical 
simulations analysis. Finally, we summarize the findings and outline some potential topics for future research.

\section{Market Model}

In this section we will consider a continuous-time, Markov regime switching financial market model consisting of a bank account and two risky assets (e.g., stocks or mutual funds). These assets are tradable continuously over a finite time horizon $[0, T]$, where $T \in(0, \infty)$. Denote the time horizon $[0, T]$ by $\mathscr{T}$. Same as [32], the standard assumptions of financial market hold, such as no transaction costs, infinitely divisible asset, and information symmetric.

A triple $(\Omega, \mathscr{F}, \mathscr{P})$ is a probability space where $\Omega$ is a set, $\mathscr{F}$ is a $\sigma$-field of subsets of $\Omega$, and $\mathscr{P}$ is a real world probability measure on $\mathscr{F}$. A subset $N$ of $\Omega$ is negligible if there exists $B \in \mathscr{F}$ such that $N \subset B$ and $\mathscr{P}(B)=0$. The probability space is complete if $\mathscr{F}$ contains the set of all negligible sets. To model uncertainties that emerged in our model, we adopt a complete probability space with filtration $\left(\Omega, \mathscr{F},\left\{\mathscr{F}_{t}\right\}_{0 \leq t \leq T}, \mathscr{P}\right)$, where $\mathscr{F}=\mathscr{F}_{T}$ and $\left\{\mathscr{F}_{t}\right\}$ describes the flow of information available to investors. We also assume the probability space is rich enough to incorporate all sources of randomness arising from fluctuations of financial asset prices and structural changes in macroeconomic conditions.

We model the evolution of the states of the economy over time by a continuous-time, finite state, time-homogeneous, observable Markov chain $\boldsymbol{\xi}:=\{\boldsymbol{\xi}(t) \mid t \in \mathscr{T}\}$ defined on $(\Omega, \mathscr{F}, \mathscr{P})$ with a finite state space $\mathcal{S}:=\left\{\mathbf{s}_{1}, \mathbf{s}_{2}, \ldots, \mathbf{s}_{N}\right\} \subset \mathfrak{R}^{N}$, where $N \geq 2$. The states of the Markov chain are interpreted as proxies of different observable macroeconomic indicators, such as gross domestic product (GDP), sovereign credit ratings, and consumer price index (CPI). More precisely, we suppose that the Markov chain is also right-continuous and irreducible.

Without loss of generality, following the convention of [33], we identify the state space of the chain as a finite set of unit basis vectors $\mathbf{E}:=\left\{\mathbf{e}_{1}, \mathbf{e}_{2}, \ldots, \mathbf{e}_{N}\right\}$, where $\mathbf{e}_{i} \in \mathfrak{R}^{N}$ and the $j$ th component of $\mathbf{e}_{i}$ is the Kronecker delta, denoted by $\delta_{i j}$, for each $i, j=1,2, \ldots, N$. Kronecker delta $\delta_{i j}$ is a piecewise function of variables $i$ and $j$ where $\delta_{i j}=1$ if $i=j$; otherwise it is zero. The set $\mathbf{E}$ is called the canonical representation of the state space of the Markov chain $\xi$ and it provides a mathematically convenient way to represent the state space of the chain. Here " $\boldsymbol{\xi}(t)=\mathbf{e}_{i}$ " means that macroeconomic indicators are in state $i$ at time $t$.

To specify the statistics properties or the probability law of the Markov chain, we define stationary transition probabilities $P_{i j}(t)=\mathscr{P}\left(\boldsymbol{\xi}(t)=\mathbf{e}_{j} \mid \boldsymbol{\xi}(0)=\mathbf{e}_{i}\right)$, for $i, j=$ $1,2, \ldots, N$ and $t \geq 0$, initial distribution $p_{i}=\mathscr{P}\left(\boldsymbol{\xi}(0)=\mathbf{e}_{i}\right)$, and the generator $Q:=\left[q_{i j}\right]_{i, j=1,2, \ldots, N}$ of the chain $\xi$ under $\mathscr{P}$ as follows:

$$
q_{i j}=\left\{\begin{array}{ll}
\lim _{t \rightarrow 0^{+}} \frac{P_{i j}(t)}{t} & i \neq j, \\
\lim _{t \rightarrow 0^{+}} \frac{P_{i i}(t)-1}{t} & i=j,
\end{array} \quad i, j=1,2, \ldots, N .\right.
$$

The generator $Q$ is also called a rate matrix or a $Q$-matrix. Here for each $i, j=1,2, \ldots, N, q_{i j}$ is the constant, instantaneous intensity of the transition of the chain $\xi$ from state $\mathbf{e}_{i}$ to $\mathbf{e}_{j}$. Note that $q_{i j} \geq 0$, for $i \neq j$ and $\sum_{j=1}^{N} q_{i j}=0$, so $q_{i i} \leq 0$. Here for each $i, j=1,2, \ldots, N$ with $i \neq j$, we assume that $q_{i j}>0$. So we obtain that $q_{i i}<0$. Then, with the canonical representation of the state space of the chain, Elliott et al. [33] provided the following semimartingale dynamic decomposition for $\xi$ :

$$
\boldsymbol{\xi}(t)=\boldsymbol{\xi}(0)+\int_{0}^{t} Q^{\prime} \boldsymbol{\xi}(u) d u+\mathbf{M}(t),
$$

where ' denotes the transpose of a matrix or a vector. Here $\{\mathbf{M}(t) \mid t \in \mathscr{T}\}$ is an $\mathfrak{R}^{N}$-valued martingale with respect to the filtration generated by Markov chain $\xi$. The filtration $\mathscr{F}^{\xi}:=\left\{\mathscr{F}^{\xi}(t) \mid t \in \mathscr{T}\right\}$ satisfies the usual conditions which are the right-continuous, $\mathscr{P}$-completed natural filtration.

In what follows, we will specify the price processes of the primitive securities and describe how the state of the economy represented by the chain $\xi$ influences the price processes. Note that the state space of the chain $\xi$ is a set of unit basis vectors, so any function of Markov chain $\boldsymbol{\xi}(t)$ can be denoted by a scalar product between a vector and $\xi(t)$.

Suppose $r(t)$ denote the instantaneous, continuously compounded interest rate of the bank account at time $t$ for each $t \in \mathscr{T}$. Then the chain determines $r(t)$ as

$$
r(t):=r(t, \boldsymbol{\xi}(t))=\langle\mathbf{r}, \boldsymbol{\xi}(t)\rangle,
$$

where $\langle\cdot, \cdot\rangle$ is the inner product in $\mathfrak{R}^{N}$ and $\mathbf{r}:=$ $\left(r_{1}, r_{2}, \ldots, r_{N}\right)^{\prime} \in \mathfrak{R}^{N}$ with $r_{i}>0$ for each $i=1,2, \ldots, N$. $r_{i}$ is interpreted as the interest rate of the bank account when the economy is in the $i$ th state. Here the inner product is to decide which component of the vectors of interest rate $\mathbf{r}$, drift rate $\boldsymbol{\mu}$, or volatility rate $\boldsymbol{\sigma}$ is in force according to the state of the economy described by the chain $\xi(t)$ at a particular time. Then the price process of the bank account $B:=\{B(t) \mid t \in$ $\mathscr{T}$ \} evolves over time according to

$$
d B(t)=r(t) B(t) d t, \quad t \in \mathscr{T}, B(0)=1 .
$$

For each $t \in \mathscr{T}$ and each $k=1,2$, suppose $\mu_{k}(t), \sigma_{k}(t)$ denote the appreciation rate and the volatility rate of the $k$ th risky asset at time $t$, respectively. Then, the chain $\xi$ also determines the appreciation rate and volatility rate of the $k$ th risky asset as

$$
\begin{aligned}
& \mu_{k}(t):=\mu_{k}(t, \boldsymbol{\xi}(t))=\left\langle\boldsymbol{\mu}_{k}, \boldsymbol{\xi}(t)\right\rangle, \\
& \sigma_{k}(t):=\sigma_{k}(t, \boldsymbol{\xi}(t))=\left\langle\boldsymbol{\sigma}_{k}, \boldsymbol{\xi}(t)\right\rangle,
\end{aligned}
$$

where $\boldsymbol{\mu}_{k}:=\left(\mu_{k}^{1}, \mu_{k}^{2}, \ldots, \mu_{k}^{N}\right)^{\prime} \in \mathfrak{R}^{N}, \sigma_{k}:=\left(\sigma_{k}^{1}, \sigma_{k}^{2}, \ldots, \sigma_{k}^{N}\right)^{\prime}$ $\in \mathfrak{R}^{N}, \mu_{k}^{i}>0$ and $\sigma_{k}^{i}>0$, for each $i=1,2, \ldots, N \cdot \mu_{k}^{i}$ and $\sigma_{k}^{i}$ are the appreciation rate and the volatility rate of the $k$ th risky asset at time $t$, respectively when the economy is in the $i$ th state at that time. Furthermore, we suppose that $\mu_{k}^{i} \geq r_{i}$ for $i=$ $1,2, \ldots, N$ and that $\mu_{k}^{i}$ 's and $\sigma_{k}^{i}$ 's are all distinct. The condition $\mu_{k}^{i} \geq r_{i}$ is necessary to exclude the arbitrage opportunities in the market. 
We consider two standard Brownian motions $W_{1}(t)$ and $W_{2}(t)$ for $t \in \mathscr{T}$ on $(\Omega, \mathscr{F}, \mathscr{P})$. To allow for complete generality, we allow the two standard Brownian motions to be correlated, with correlation coefficient denoted by $\rho$; namely, $E\left[W_{1}(t) W_{2}(t)\right]=\rho t$. In case there would only be one source of randomness left in the model, we also assume that $\rho^{2}<1$. At the same time we also assume the two standard Brownian motions are independent of the Markov chain $\xi$. For each $k=1,2$, let $S_{k}:=\left\{S_{k}(t) \mid t \in \mathscr{T}\right\}$ denote the price process of the $k$ th risky asset. Then, we assume that the evolution of $S_{k}$ over time satisfies the following Markov regime switching geometric Brownian motion:

$$
\begin{gathered}
d S_{k}(t)=\mu_{k}(t) S_{k}(t) d t+\sigma_{k}(t) S_{k}(t) d W_{k}(t), \\
S_{k}(0)=s_{0},
\end{gathered}
$$

where the market price of risk for risky asset $k$ is defined as $\theta_{k}(t)=\left(\mu_{k}(t)-r(t)\right) / \sigma_{k}(t)$.

We consider stochastic dynamic portfolio game in a continuous-time financial market between two investors; call them A and B. Without loss of generality, we suppose that there is a bank account that is freely available to both investors and simultaneously, there are only two correlated risky assets in the financial market, only one of which is available to each investor. Investor A may be allowed to trade in the first risky asset, $S_{1}$, and similarly, investor B may be restricted to trade only in the second risky asset, $S_{2}$. They cooperate with each other on investment by the choice of their individual dynamic portfolio trading strategies in the risky assets and bank account.

In the next we describe the wealth dynamic processes of both investors. For each $t \in \mathscr{T}$, let $X\left(t, \pi_{1}\right)$ denote the wealth process of investor A at time $t$ under a portfolio strategy $\pi_{1}:=\left\{\pi_{1}(t) \mid t \in \mathscr{T}\right\}$ with $X(0)=x_{0}$. Investor $\mathrm{A}$ invests an amount of wealth $\pi_{1}(t)$ in the risky asset $S_{1}$ at time $t$. Note that once $\pi_{1}(t)$ is determined, the remaining amount invested in the bank account is completely specified as $X\left(t, \pi_{1}\right)-\pi_{1}(t)$. Similarly, let $Y\left(t, \pi_{2}\right)$ denote the wealth process of investor B at time $t$ under a portfolio strategy $\pi_{2}:=\left\{\pi_{2}(t) \mid t \in \mathscr{T}\right\}$ with $Y(0)=y_{0}$. Investor B invests an amount of wealth $\pi_{2}(t)$ in the risky asset $S_{2}$ at time $t$. The remaining amount $Y\left(t, \pi_{2}\right)-\pi_{2}(t)$ is in the bank account.

Let $\Pi_{1}$ be the space of all admissible portfolio strategies $\pi_{1}$. The elements in $\Pi_{1}$ satisfy the following two conditions: (i) $\mathscr{F}$-progressively measurable and càdlàg (right-continuous with left limit) $\mathfrak{R}$-valued process (i.e., $\pi_{1}$ is a nonanticipative function) and (ii) $E\left[\int_{0}^{T} \pi_{1}^{2}(t) d t\right]<\infty$. The condition (ii) is a technical condition. If $\pi_{1} \in \Pi_{1}$, we call the portfolio strategy $\pi_{1}$ admissible. So $\Pi_{1}$ is the set of all admissible portfolio strategies of investor A. Similarly, we can define the set of all admissible portfolio strategies of investor B and denote it by $\Pi_{2}$.

As in a standard portfolio selection problem, the portfolio strategies (controls) are assumed to be piecewise continuous. We also assume the portfolio strategies of stochastic differential game between the two investors are feedback strategies, more specifically, Markov control strategies. Markov control is only dependent on the current value of state variables in the system not upon the history. That is, the value we choose at time $t$ only depends on the state of the system at this time. Furthermore, the investor can condition his action at each point in time on the basis of the state of the system at that point in time. In many cases, it suffices to consider Markov control. For more discussions on the strategies employed in the differential games, interested readers can refer to [34].

We place no other restrictions on portfolio strategy $\pi_{1}$ or $\pi_{2}$. For example, we allow $\pi_{1}(t)<0$ or $\left(\pi_{2}(t)<0\right)$; this means the investors are allowed to sell the risky assets short. Whereas we allow $\pi_{1}(t)>X\left(t, \pi_{1}\right)$ or $\left(\pi_{2}(t)>X\left(t, \pi_{2}\right)\right)$, this corresponds to a credit and it means the investors have borrowed to purchase the risky assets. Here, we note that the investor decides the wealth amount allocated to the risky asset according to the current and past market prices information and observations of market or macroeconomic conditions. This is totally different from some traditional optimal portfolio models, where the investors only consider the price information in making their optimal investment decisions.

Under the self-financing assumption, for each $t \in \mathscr{T}$ the dynamics of the wealth process $X\left(t, \pi_{1}\right)$ associated with $\pi_{1}$ of investor A evolves over time as the following Markov regime switching stochastic differential equation:

$$
\begin{aligned}
& d X\left(t, \pi_{1}\right) \\
& =\left[r(t, \xi(t)) X\left(t, \pi_{1}\right)+\left(\mu_{1}(t, \xi(t))-r(t, \xi(t))\right) \pi_{1}(t)\right] d t \\
& \quad+\sigma_{1}(t, \xi(t)) \pi_{1}(t) d W_{1}(t) .
\end{aligned}
$$

Similarly, for each $t \in \mathscr{T}$ the dynamics of the wealth process $Y\left(t, \pi_{2}\right)$ associated with $\pi_{2}$ of investor B is governed by the following Markov-modulated stochastic differential equation:

$$
\begin{aligned}
& d Y\left(t, \pi_{2}\right) \\
& =\left[r(t, \xi(t)) Y\left(t, \pi_{2}\right)+\left(\mu_{2}(t, \xi(t))-r(t, \xi(t))\right) \pi_{2}(t)\right] d t \\
& \quad+\sigma_{2}(t, \xi(t)) \pi_{2}(t) d W_{2}(t) .
\end{aligned}
$$

For each $t \in \mathscr{T}$, denote the sum of wealth processes by $Z^{\left(\pi_{1}, \pi_{2}\right)}(t)=X\left(t, \pi_{1}\right)+Y\left(t, \pi_{2}\right)$. Since $X\left(t, \pi_{1}\right)$ and $Y\left(t, \pi_{2}\right)$ are diffusion processes controlled by investors $\mathrm{A}$ and $\mathrm{B}$, respectively, then $Z^{\left(\pi_{1}, \pi_{2}\right)}(t)$ is a jointly controlled diffusion process. Specifically, the evolution of the sum process over time is governed by the following Markov regime switching stochastic differential equation:

$$
\begin{aligned}
& d Z^{\left(\pi_{1}, \pi_{2}\right)}(t) \\
& =\left[r(t, \xi(t)) Z^{\left(\pi_{1}, \pi_{2}\right)}(t)+\left(\mu_{1}(t, \xi(t))-r(t, \xi(t))\right) \pi_{1}(t)\right. \\
& \left.\quad+\left(\mu_{2}(t, \xi(t))-r(t, \xi(t))\right) \pi_{2}(t)\right] d t \\
& \quad+\sigma_{1}(t, \xi(t)) \pi_{1}(t) d W_{1}(t)+\sigma_{2}(t, \xi(t)) \pi_{2}(t) d W_{2}(t),
\end{aligned}
$$

where $Z^{\left(\pi_{1}, \pi_{2}\right)}(0)=x_{0}+y_{0}$. 
For mathematical convenience, we can rewrite (9) in a more compact form

$$
\begin{aligned}
d Z^{\left(\pi_{1}, \pi_{2}\right)}(t)=[ & r(t) Z^{\left(\pi_{1}, \pi_{2}\right)}(t)+\left(\mu_{1}(t)-r(t)\right) \pi_{1}(t) \\
& \left.+\left(\mu_{2}(t)-r(t)\right) \pi_{2}(t)\right] d t \\
& +\sigma_{1}(t) \pi_{1}(t) d W_{1}(t)+\sigma_{2}(t) \pi_{2}(t) d W_{2}(t) .
\end{aligned}
$$

In the next section we provide a utility-based stochastic differential portfolio game with respect to the process $\left\{Z^{\left(\pi_{1}, \pi_{2}\right)}, t \in \mathscr{T}\right\}$ of (9) or (10). We formulate the stochastic differential portfolio game as a problem of maximizing the expected utility of the sum of terminal wealth processes. More general results on zero-sum stochastic differential portfolio games are discussed in, for example, Browne [20]. Moreover, for some results on nonzero-sum differential games, interested readers can refer to Lin [29].

\section{Nonzero-Sum Game Problem Formulation}

In this section, we consider nonzero-sum stochastic differential portfolio game problem between two investors. The differential game is formulated as a problem to maximizing expected utility of the sum of terminal wealth processes of two investors, respectively, at some fixed time $T \in \mathscr{T}$.

For each $k=1,2$, let $U_{k}: \mathfrak{R}^{+} \rightarrow \mathfrak{R}$ denote utility functions of investors $\mathrm{A}$ and $\mathrm{B}$, respectively, which are both strictly increasing, strictly concave, and continuous differentiable (i.e., $U_{k}^{\prime}>0$ and $U_{k}^{\prime \prime}<0$ ). More results about risk preference can refer to [35-37]. Furthermore, we assume that the utility functions satisfy the following Inada conditions (technical conditions):

$$
\begin{aligned}
& U_{k}^{\prime}(0+)=\lim _{z \rightarrow 0^{+}} U_{k}^{\prime}(z)=+\infty, \\
& U_{k}^{\prime}(+\infty)=\lim _{z \rightarrow+\infty} U_{k}^{\prime}(z)=0 .
\end{aligned}
$$

In the case of two investors, $\mathrm{A}$ and $\mathrm{B}$, for each $t \in \mathscr{T}$ and each $i=1,2, \ldots, N$, a typical differential game is posed as follows. Given $\boldsymbol{\xi}(t)=\mathbf{e}_{i}$ and $Z^{\left(\pi_{1}, \pi_{2}\right)}(t)=z$, investor A choose his own admissible portfolio strategy $\pi_{1} \in \Pi_{1}$ to maximize

$$
\begin{aligned}
& V_{1}^{\left(\pi_{1}, \pi_{2}\right)}\left(t, z, \mathbf{e}_{i}\right) \\
& \quad=E\left[U_{1}\left[Z^{\left(\pi_{1}, \pi_{2}\right)}(T)\right] \mid Z^{\left(\pi_{1}, \pi_{2}\right)}(t)=z, \xi(t)=\mathbf{e}_{i}\right],
\end{aligned}
$$

while investor B choose his own admissible portfolio strategy $\pi_{2} \in \Pi_{2}$ to maximize

$$
\begin{aligned}
& V_{2}^{\left(\pi_{1}, \pi_{2}\right)}\left(t, z, \mathbf{e}_{i}\right) \\
& \quad=E\left[U_{2}\left[Z^{\left(\pi_{1}, \pi_{2}\right)}(T)\right] \mid Z^{\left(\pi_{1}, \pi_{2}\right)}(t)=z, \boldsymbol{\xi}(t)=\mathbf{e}_{i}\right],
\end{aligned}
$$

with both utility maximization problems subject to the sum process (9) or (10).

We assume that each investor is aware of the other investor's presence and how the other's choice of his strategy affects the state equation. Furthermore, we assume that the two investors choose their portfolio strategies simultaneously. Investor A would like to choose an admissible strategy $\pi_{1}$ so as to maximize his payoff $V_{1}^{\left(\pi_{1}, \pi_{2}\right)}\left(t, z, \mathbf{e}_{i}\right)$ for every possible choice of investor B's portfolio strategy, while investor B is trying to choose an admissible strategy $\pi_{2}$ in order to maximize his payoff $V_{2}^{\left(\pi_{1}, \pi_{2}\right)}\left(t, z, \mathbf{e}_{i}\right)$ for every possible choice of investor A's portfolio strategy. The game terminates at a fixed duration $T$. Then the nonzero-sum stochastic differential portfolio game can be formulated as the following two optimal portfolio selection utility maximization problems of investors $\mathrm{A}$ and $\mathrm{B}$ :

$$
\begin{aligned}
& V_{1}\left(t, z, \mathbf{e}_{i}\right):=\sup _{\pi_{1} \in \Pi_{1}} V_{1}^{\left(\pi_{1}, \pi_{2}^{*}\right)}\left(t, z, \mathbf{e}_{i}\right), \\
& V_{2}\left(t, z, \mathbf{e}_{i}\right):=\sup _{\pi_{2} \in \Pi_{2}} V_{2}^{\left(\pi_{1}^{*}, \pi_{2}\right)}\left(t, z, \mathbf{e}_{i}\right) .
\end{aligned}
$$

Here $V_{1}\left(t, z, \mathbf{e}_{i}\right)$ and $V_{2}\left(t, z, \mathbf{e}_{i}\right)$ are the value functions of the optimal portfolio selection problems associated with investors $\mathrm{A}$ and $\mathrm{B}$, respectively, over the time horizon $[t, T]$. This is a two-player, nonzero-sum, stochastic differential portfolio game between two investors $\mathrm{A}$ and $\mathrm{B}$.

To solve the nonzero-sum stochastic differential portfolio game, in the following, we first give the definition of Nash equilibrium for the differential game between two investors $A$ and $B$ described above.

Definition 1. For each time $t \in \mathscr{T}$, given that the state of macroeconomic is in the $i$ th state, let $\pi_{2}$ be an admissible strategy of investor $\mathrm{B}$. One defines the set of best responses of investor A to the admissible portfolio strategy $\pi_{2}$ as

$$
\begin{aligned}
B R_{1}^{i}\left(\pi_{2}\right) & \\
& :=\left\{\pi_{1}^{*} \in \Pi_{1} \mid V_{1}^{\left(\pi_{1}^{*}, \pi_{2}\right)}\left(t, z, \mathbf{e}_{i}\right)=\sup _{\pi_{1} \in \Pi_{1}} V_{1}^{\left(\pi_{1}, \pi_{2}\right)}\left(t, z, \mathbf{e}_{i}\right)\right\} .
\end{aligned}
$$

And similarly, one can define the set of the best responses of investor B to the strategy $\pi_{1}$ of investor $A$ as

$$
\begin{aligned}
B_{2}^{i}\left(\pi_{1}\right) & \\
& :=\left\{\pi_{2}^{*} \in \Pi_{2} \mid V_{2}^{\left(\pi_{1}, \pi_{2}^{*}\right)}\left(t, z, \mathbf{e}_{i}\right)=\sup _{\pi_{2} \in \Pi_{2}} V_{2}^{\left(\pi_{1}, \pi_{2}\right)}\left(t, z, \mathbf{e}_{i}\right)\right\} .
\end{aligned}
$$

A pair of admissible portfolio strategies $\left(\pi_{1}^{*}, \pi_{2}^{*}\right)$ is said to be a Nash equilibrium (i.e., saddle point) for the nonzero-sum differential game with investors $A$ and $B$, strategies spaces $\Pi_{1}$ 
and $\Pi_{2}$, and payoffs (12) and (13) when the economy (Markov chain) is in state $\mathbf{e}_{i}$ if

$$
\pi_{1}^{*} \in B R_{1}^{i}\left(\pi_{2}^{*}\right), \quad \pi_{2}^{*} \in B R_{2}^{i}\left(\pi_{1}^{*}\right)
$$

Equivalently, $\left(\pi_{1}^{*}, \pi_{2}^{*}\right)$ is said to be a Nash equilibrium if

$$
\begin{aligned}
& V_{1}^{\left(\pi_{1}^{*}, \pi_{2}^{*}\right)}\left(t, z, \mathbf{e}_{i}\right)=\sup _{\pi_{1} \in \Pi_{1}} V_{1}^{\left(\pi_{1}, \pi_{2}^{*}\right)}\left(t, z, \mathbf{e}_{i}\right), \\
& V_{2}^{\left(\pi_{1}^{*}, \pi_{2}^{*}\right)}\left(t, z, \mathbf{e}_{i}\right)=\sup _{\pi_{2} \in \Pi_{2}} V_{2}^{\left(\pi_{1}^{*}, \pi_{2}\right)}\left(t, z, \mathbf{e}_{i}\right),
\end{aligned}
$$

where $\pi_{1}^{*}$ and $\pi_{2}^{*}$ are referred to as investor A's and investor B's respective equilibrium strategies. If a Nash equilibrium exists, then the value functions of the nonzero-sum differential game can be obtained. For more discussions on the implications of Nash equilibrium, interested readers can refer to [38].

\section{Regime Switching HJB Equation and the Optimal Conditions}

In this section we will derive a pair of regime switching $\mathrm{HJB}$ equations and Feynman-Kac representations for the value functions of the nonzero-sum differential game formulated in the last section. We will also derive a set of coupled $\mathrm{HJB}$ equations corresponding to the regime switching HJB equations.

In the sequel, we consider the case of investors with risk averse exponential utility functions. Suppose that utility functions $U_{1}$ of investor $\mathrm{A}$ and $U_{2}$ of investor $\mathrm{B}$ are given by

$$
U_{1}(x)=-\frac{1}{\gamma_{1}} e^{-\gamma_{1} x}, \quad U_{2}(x)=-\frac{1}{\gamma_{2}} e^{-\gamma_{2} x},
$$

where $\gamma_{1}$ and $\gamma_{2}$ are positive constants, which represent the coefficients of absolute risk aversion (CARA) of investors. That is,

$$
\gamma_{k}=-\frac{U_{k}^{\prime \prime}(x)}{U_{k}^{\prime}(x)}, \quad k=1,2
$$

with $U_{k}^{\prime}(x)$ and $U_{k}^{\prime \prime}(x)$ representing the first and second derivatives of $U_{k}$ with respect to $x$, for each $k=1,2$.

Let $V_{k}^{i}:=V_{k}\left(t, z, \mathbf{e}_{i}\right)$ and $\mathbf{V}_{k}:=\left(V_{k}^{1}, V_{k}^{2}, \ldots, V_{k}^{N}\right)$, for each $k=1,2$ and each $i=1,2, \ldots, N$. We now solve the two utility maximization problems via the dynamic programming principle in stochastic optimal control according to [39]. It can be shown that value functions $\mathbf{V}_{k}$ of utility maximization of the nonzero-sum differential game satisfy the following regime switching HJB equations:

$$
\begin{aligned}
& \frac{\partial \mathbf{V}_{1}}{\partial t}+\sup _{\pi_{1} \in \Pi_{1}}\left\{\left[r(t) z+\left(\mu_{1}(t)-r(t)\right) \pi_{1}(t)\right.\right. \\
& \left.+\left(\mu_{2}(t)-r(t)\right) \pi_{2}^{*}(t)\right] \frac{\partial \mathbf{V}_{1}}{\partial z} \\
& +\frac{1}{2}\left[\sigma_{1}^{2}(t) \pi_{1}^{2}(t)+\sigma_{2}^{2}(t)\left(\pi_{2}^{*}(t)\right)^{2}\right. \\
& \left.+2 \rho \sigma_{1}(t) \sigma_{2}(t) \pi_{1}(t) \pi_{2}^{*}(t)\right] \frac{\partial^{2} \mathbf{V}_{1}}{\partial z^{2}} \\
& \left.+\left\langle\mathbf{V}_{1}, Q^{\prime} \xi(t)\right\rangle\right\}=0 \\
& \frac{\partial \mathbf{V}_{2}}{\partial t}+\sup _{\pi_{2} \in \Pi_{2}}\left\{\left[r(t) z+\left(\mu_{1}(t)-r(t)\right) \pi_{1}^{*}(t)\right.\right. \\
& \left.+\left(\mu_{2}(t)-r(t)\right) \pi_{2}(t)\right] \frac{\partial \mathbf{V}_{2}}{\partial z} \\
& +\frac{1}{2}\left[\sigma_{1}^{2}(t)\left(\pi_{1}^{*}(t)\right)^{2}+\sigma_{2}^{2}(t) \pi_{2}^{2}(t)\right. \\
& \left.+2 \rho \sigma_{1}(t) \sigma_{2}(t) \pi_{1}^{*}(t) \pi_{2}(t)\right] \frac{\partial^{2} \mathbf{V}_{2}}{\partial z^{2}} \\
& \left.+\left\langle\mathbf{V}_{2}, Q^{\prime} \xi(t)\right\rangle\right\}=0
\end{aligned}
$$

In what follows of this section, with a slight abuse of notations, for each $k=1,2$, we still let all the notations $r\left(t, \mathbf{e}_{i}\right), \mu_{k}\left(t, \mathbf{e}_{i}\right), \sigma_{k}\left(t, \mathbf{e}_{i}\right)$, and $\pi_{k}\left(t, \mathbf{e}_{i}\right)$ be denoted by $r(t)$, $\mu_{k}(t), \sigma_{k}(t)$, and $\pi_{k}(t)$ for $t \in \mathscr{T}$, unless otherwise stated. For each $i=1,2, \ldots, N$, let $V_{k}^{i}:=V_{k}\left(t, z, e_{i}\right)$. Hence, the vector $\mathbf{V}_{k}$ of value functions at different regimes satisfies the following two systems of coupled regime switching HJB equations, respectively:

$$
\begin{aligned}
\frac{\partial V_{1}^{i}}{\partial t}+\sup _{\pi_{1} \in \Pi_{1}}\{[ & r(t) z+\left(\mu_{1}(t)-r(t)\right) \pi_{1}(t) \\
& \left.+\left(\mu_{2}(t)-r(t)\right) \pi_{2}^{*}(t)\right] \frac{\partial V_{1}^{i}}{\partial z} \\
& +\frac{1}{2}\left[\sigma_{1}^{2}(t) \pi_{1}^{2}(t)+\sigma_{2}^{2}(t)\left(\pi_{2}^{*}(t)\right)^{2}\right. \\
& \left.+2 \rho \sigma_{1}(t) \sigma_{2}(t) \pi_{1}(t) \pi_{2}^{*}(t)\right] \frac{\partial^{2} V_{1}^{i}}{\partial z^{2}} \\
& \left.+\sum_{j \in E} q_{i j}\left[V_{1}\left(t, z, \mathbf{e}_{j}\right)-V_{1}\left(t, z, \mathbf{e}_{i}\right)\right]\right\}=0,
\end{aligned}
$$


with the terminal condition $V_{1}\left(T, z, \mathbf{e}_{i}\right)=U_{1}(z)=$ $-\left(1 / \gamma_{1}\right) e^{-\gamma_{1} z}$

$$
\begin{aligned}
\frac{\partial V_{2}^{i}}{\partial t}+\sup _{\pi_{2} \in \Pi_{2}}\{[ & r(t) z+\left(\mu_{1}(t)-r(t)\right) \pi_{1}^{*}(t) \\
& \left.+\left(\mu_{2}(t)-r(t)\right) \pi_{2}(t)\right] \frac{\partial V_{2}^{i}}{\partial z} \\
+ & \frac{1}{2}\left[\sigma_{1}^{2}(t)\left(\pi_{1}^{*}(t)\right)^{2}+\sigma_{2}^{2}(t) \pi_{2}^{2}(t)\right. \\
& \left.+2 \rho \sigma_{1}(t) \sigma_{2}(t) \pi_{1}^{*}(t) \pi_{2}(t)\right] \frac{\partial^{2} V_{2}^{i}}{\partial z^{2}} \\
& \left.+\sum_{j \in E} q_{i j}\left[V_{2}\left(t, z, \mathbf{e}_{j}\right)-V_{2}\left(t, z, \mathbf{e}_{i}\right)\right]\right\}=0,
\end{aligned}
$$

with the terminal condition $V_{2}\left(T, z, \mathbf{e}_{i}\right)=U_{2}(z)=$ $-\left(1 / \gamma_{2}\right) e^{-\gamma_{2} z}$.

In what follows, to abbreviate the expression in curly brackets, for each $k=1,2$, we define the operators

$$
\begin{aligned}
& \mathscr{L}^{\left(\pi_{1}, \pi_{2}\right)} V_{k}\left(t, z, \mathbf{e}_{i}\right) \\
& =\left[r(t) z+\left(\mu_{1}(t)-r(t)\right) \pi_{1}(t)+\left(\mu_{2}(t)-r(t)\right) \pi_{2}(t)\right] \frac{\partial V_{k}^{i}}{\partial z} \\
& \quad+\frac{1}{2}\left[\sigma_{1}^{2}(t) \pi_{1}^{2}(t)+\sigma_{2}^{2}(t) \pi_{2}^{2}(t)\right. \\
& \left.\quad+2 \rho \sigma_{1}(t) \sigma_{2}(t) \pi_{1}(t) \pi_{2}(t)\right] \frac{\partial^{2} V_{k}^{i}}{\partial z^{2}} \\
& +\sum_{j \in E} q_{i j}\left[V_{k}\left(t, z, \mathbf{e}_{j}\right)-V_{k}\left(t, z, \mathbf{e}_{i}\right)\right], \quad i=1,2, \ldots, N .
\end{aligned}
$$

Then the HJB equations (22) and (23) can be simplified as follows:

$$
\begin{aligned}
& \frac{\partial V_{1}^{i}}{\partial t}+\sup _{\pi_{1} \in \Pi_{1}} \mathscr{L}^{\left(\pi_{1}, \pi_{2}^{*}\right)} V_{1}\left(t, z, \mathbf{e}_{i}\right)=0, \\
& \frac{\partial V_{2}^{i}}{\partial t}+\sup _{\pi_{2} \in \Pi_{2}} \mathscr{L}^{\left(\pi_{1}^{*}, \pi_{2}\right)} V_{2}\left(t, z, \mathbf{e}_{i}\right)=0 .
\end{aligned}
$$

First, the first-order conditions for maximizing the quantity in the HJB equations (25) and (26) give optimal portfolio strategies

$$
\begin{array}{r}
\pi_{1}^{*}(t)=\frac{1}{1-\rho^{2}}\left(\rho \frac{\mu_{2}(t)-r(t)}{\sigma_{1}(t) \sigma_{2}(t)} \frac{\partial V_{2}^{i} / \partial z}{\partial^{2} V_{2}^{i} / \partial z^{2}}\right. \\
\left.-\frac{\mu_{1}(t)-r(t)}{\sigma_{1}^{2}(t)} \frac{\partial V_{1}^{i} / \partial z}{\partial^{2} V_{1}^{i} / \partial z^{2}}\right), \\
\pi_{2}^{*}(t)=\frac{1}{1-\rho^{2}}\left(\rho \frac{\mu_{1}(t)-r(t)}{\sigma_{1}(t) \sigma_{2}(t)} \frac{\partial V_{1}^{i} / \partial z}{\partial^{2} V_{1}^{i} / \partial z^{2}}\right. \\
\left.-\frac{\mu_{2}(t)-r(t)}{\sigma_{2}^{2}(t)} \frac{\partial V_{2}^{i} / \partial z}{\partial^{2} V_{2}^{i} / \partial z^{2}}\right) .
\end{array}
$$

Second, assume that HJB equations (22) or (25) and (23) or (26) have smooth solutions with $V_{k, z}^{i}>0$ and $V_{k, z z}^{i}<0$ for each $k=1,2$. The subscripts on $V_{k, z}^{i}$ and $V_{k, z z}^{i}$ denote the first and second partial differentiation with respect to variable $z$, respectively. Following the approach in [3], then we consider value functions are of the following trial solutions:

$$
V_{1}^{i}=V_{1}\left(t, z, \mathbf{e}_{i}\right)=-\frac{1}{\gamma_{1}} e^{-\gamma_{1} z e^{\int_{t}^{T} r(s) d s}} f\left(t, \mathbf{e}_{i}\right),
$$

where $f\left(t, \mathbf{e}_{i}\right) \in \mathscr{C}^{1}$, to be determined, is a suitable positive function with the boundary condition $f\left(T, \mathbf{e}_{i}\right)=1$ for all $\mathbf{e}_{i} \in$ E. Consider

$$
V_{2}^{i}=V_{2}\left(t, z, \mathbf{e}_{i}\right)=-\frac{1}{\gamma_{2}} e^{-\gamma_{2} z e^{\int_{t}^{T} r(s) d s}} g\left(t, \mathbf{e}_{i}\right),
$$

where $g\left(t, \mathbf{e}_{i}\right) \in \mathscr{C}^{1}$, to be determined, is a suitable positive function with the boundary condition $g\left(T, \mathbf{e}_{i}\right)=1$ for all $\mathbf{e}_{i} \in$ E.

Hence, from (27) we obtain the associated explicit expressions of competitive optimal portfolio strategies

$$
\begin{aligned}
& \pi_{1}^{*}(t)=\frac{1}{1-\rho^{2}}\left(\frac{\mu_{1}(t)-r(t)}{\gamma_{1} \sigma_{1}^{2}(t)} e^{-\int_{t}^{T} r(s) d s}\right. \\
& \left.-\rho \frac{\mu_{2}(t)-r(t)}{\gamma_{2} \sigma_{1}(t) \sigma_{2}(t)} e^{-\int_{t}^{T} r(s) d s}\right), \\
& \pi_{2}^{*}(t)=\frac{1}{1-\rho^{2}}\left(\frac{\mu_{2}(t)-r(t)}{\gamma_{2} \sigma_{2}^{2}(t)} e^{-\int_{t}^{T} r(s) d s}\right.
\end{aligned}
$$


Last, substituting these results into HJB equations (22) or (25) and (23) or (26), we derive the following two systems of coupled linear ordinary differential equations:

$$
\begin{aligned}
& \frac{\partial f\left(t, \mathbf{e}_{i}\right)}{\partial t}+\frac{\gamma_{1}^{2}}{2\left(1-\rho^{2}\right)} \\
& \cdot\left[\frac{\left(\mu_{2}(t)-r(t)\right)^{2}}{\gamma_{2}^{2} \sigma_{2}^{2}(t)}-\frac{\left(\mu_{1}(t)-r(t)\right)^{2}}{\gamma_{1}^{2} \sigma_{1}^{2}(t)}\right. \\
& -2 \frac{\left(\mu_{2}(t)-r(t)\right)^{2}}{\gamma_{1} \gamma_{2} \sigma_{2}^{2}(t)} \\
& \left.+2 \rho \frac{\left(\mu_{1}(t)-r(t)\right)\left(\mu_{2}(t)-r(t)\right)}{\gamma_{1}^{2} \sigma_{1}(t) \sigma_{2}(t)}\right] f\left(t, \mathbf{e}_{i}\right) \\
& +\sum_{j \in E} q_{i j}\left[f\left(t, \mathbf{e}_{j}\right)-f\left(t, \mathbf{e}_{i}\right)\right]=0, \quad f\left(T, \mathbf{e}_{i}\right)=1, \\
& \frac{\partial g\left(t, \mathbf{e}_{i}\right)}{\partial t}+\frac{\gamma_{2}^{2}}{2\left(1-\rho^{2}\right)} \\
& \cdot\left[\frac{\left(\mu_{1}(t)-r(t)\right)^{2}}{\gamma_{1}^{2} \sigma_{1}^{2}(t)}-\frac{\left(\mu_{2}(t)-r(t)\right)^{2}}{\gamma_{2}^{2} \sigma_{2}^{2}(t)}\right. \\
& -2 \frac{\left(\mu_{1}(t)-r(t)\right)^{2}}{\gamma_{1} \gamma_{2} \sigma_{1}^{2}(t)} \\
& \left.+2 \rho \frac{\left(\mu_{1}(t)-r(t)\right)\left(\mu_{2}(t)-r(t)\right)}{\gamma_{2}^{2} \sigma_{1}(t) \sigma_{2}(t)}\right] g\left(t, \mathbf{e}_{i}\right) \\
& +\sum_{j \in E} q_{i j}\left[g\left(t, \mathbf{e}_{j}\right)-g\left(t, \mathbf{e}_{i}\right)\right]=0, \quad g\left(T, \mathbf{e}_{i}\right)=1 .
\end{aligned}
$$

It is well known that both systems of differential equations only have a unique solution, respectively. We will give an appropriate explanation in Remark 8 of this section. In summary, we obtain the following theorem immediately.

Theorem 2. If $\rho^{2} \neq 1$, then the value functions of investors $A$ and $B$ at different regimes of the utility maximization of nonzero-sum stochastic differential portfolio game are given by

$$
\begin{aligned}
& V_{1}^{i}=V_{1}\left(t, z, \mathbf{e}_{i}\right)=-\frac{1}{\gamma_{1}} e^{-\gamma_{1} z e^{\int_{t}^{T} r(s) d s}} f\left(t, \mathbf{e}_{i}\right), \\
& V_{2}^{i}=V_{2}\left(t, z, \mathbf{e}_{i}\right)=-\frac{1}{\gamma_{2}} e^{-\gamma_{2} z e^{\int_{t}^{T} r(s) d s}} g\left(t, \mathbf{e}_{i}\right),
\end{aligned}
$$

where $f\left(t, \mathbf{e}_{i}\right)$ and $g\left(t, \mathbf{e}_{i}\right)$ are solutions of coupled linear ordinary differential equations (31) and (32). The optimal portfolio strategies can be expressed as

$$
\begin{aligned}
\pi_{1}^{*}(t)=\frac{1}{1-\rho^{2}}\left(\frac{\mu_{1}(t)-r(t)}{\gamma_{1} \sigma_{1}^{2}(t)} e^{-\int_{t}^{T} r(s) d s}\right. & \\
& \left.-\rho \frac{\mu_{2}(t)-r(t)}{\gamma_{2} \sigma_{1}(t) \sigma_{2}(t)} e^{-\int_{t}^{T} r(s) d s}\right), \\
\pi_{2}^{*}(t)=\frac{1}{1-\rho^{2}}\left(\frac{\mu_{2}(t)-r(t)}{\gamma_{2} \sigma_{2}^{2}(t)} e^{-\int_{t}^{T} r(s) d s}\right. & \left.-\rho \frac{\mu_{1}(t)-r(t)}{\gamma_{1} \sigma_{1}(t) \sigma_{2}(t)} e^{-\int_{t}^{T} r(s) d s}\right) .
\end{aligned}
$$

Remark 3. From the expressions (34) of optimal portfolio strategies, we can see that the optimal portfolio strategies are composed of two terms and the first term is similar to Merton-type solution. Meanwhile, we find that the portfolio strategies also depend on the regime switching of the macroeconomy. Both of them are directly proportional to the expected excess returns on the corresponding risky assets in the market when the correlation $\rho$ between two risky assets is negative. However, when $\rho>0$, the optimal strategies are inversely proportional to the expected excess return of the other risky asset not available to the associated investors. Furthermore, even if the macroeconomy has $N$ states, only if the state of economy is unchanged, we find that the optimal portfolio strategies are always constant. Namely, they are independent of the wealth level of investors.

Remark 4. When the Markov chain has only one state (i.e., $\mathcal{S}:=\left\{\mathbf{s}_{1}\right\}$ ), then the Markov regime switching model considered here degenerates into a deterministic case. And the market parameters $\mathbf{r}, \boldsymbol{\mu}$, and $\boldsymbol{\sigma}$ of the model become constants. Then the conclusions will not be influenced by Markov chain, so we can omit the index $i$ in the value functions and the optimal strategies. Albeit with the difference in form, the linear ordinary differential equations which the functions $f$ and $g$ in the value functions satisfy coincide with (4.3) and (4.4) of lemma 4.1 of [29] respectively. Correspondingly the value functions and optimal strategies are consistent with the results of Theorem 4.1 of [29].

Corollary 5. If $\rho=0$, the two standard Brownian motions $\left(W_{1}(t)\right.$ and $\left.W_{2}(t)\right)$ are mutual, independent of $(\Omega, \mathscr{F}, \mathscr{P})$. Then the value functions of investors $A$ and $B$ at different regimes of the utility maximization of the nonzero-sum stochastic differential portfolio game are denoted by

$$
\begin{aligned}
& V_{1}^{i}=V_{1}\left(t, z, \mathbf{e}_{i}\right)=-\frac{1}{\gamma_{1}} e^{-\gamma_{1} z e^{\int_{t}^{T} r(s) d s}} f\left(t, \mathbf{e}_{i}\right), \\
& V_{2}^{i}=V_{2}\left(t, z, \mathbf{e}_{i}\right)=-\frac{1}{\gamma_{2}} e^{-\gamma_{2} z e^{\int_{t}^{T} r(s) d s}} g\left(t, \mathbf{e}_{i}\right),
\end{aligned}
$$

and the optimal portfolio strategies are given by

$$
\begin{aligned}
& \pi_{1}^{*}(t)=\frac{\mu_{1}(t)-r(t)}{\gamma_{1} \sigma_{1}^{2}(t)} e^{-\int_{t}^{T} r(s) d s}, \\
& \pi_{2}^{*}(t)=\frac{\mu_{2}(t)-r(t)}{\gamma_{2} \sigma_{2}^{2}(t)} e^{-\int_{t}^{T} r(s) d s},
\end{aligned}
$$


where the functions $f$ and $g$ are the solutions of the following linear ordinary differential equations:

$$
\begin{gathered}
\frac{\partial f\left(t, \mathbf{e}_{i}\right)}{\partial t}+\frac{\gamma_{1}^{2}}{2}\left[\frac{\left(\mu_{2}(t)-r(t)\right)^{2}}{\gamma_{2}^{2} \sigma_{2}^{2}(t)}-\frac{\left(\mu_{1}(t)-r(t)\right)^{2}}{\gamma_{1}^{2} \sigma_{1}^{2}(t)}\right. \\
\left.-2 \frac{\left(\mu_{2}(t)-r(t)\right)^{2}}{\gamma_{1} \gamma_{2} \sigma_{2}^{2}(t)}\right] f\left(t, \mathbf{e}_{i}\right) \\
+\sum_{j \in E} q_{i j}\left[f\left(t, \mathbf{e}_{j}\right)-f\left(t, \mathbf{e}_{i}\right)\right]=0, \quad f\left(T, \mathbf{e}_{i}\right)=1,
\end{gathered}
$$

$$
\begin{gathered}
\frac{\partial g\left(t, \mathbf{e}_{i}\right)}{\partial t}+\frac{\gamma_{2}^{2}}{2}\left[\frac{\left(\mu_{1}(t)-r(t)\right)^{2}}{\gamma_{1}^{2} \sigma_{1}^{2}(t)}-\frac{\left(\mu_{2}(t)-r(t)\right)^{2}}{\gamma_{2}^{2} \sigma_{2}^{2}(t)}\right. \\
\left.-2 \frac{\left(\mu_{1}(t)-r(t)\right)^{2}}{\gamma_{1} \gamma_{2} \sigma_{1}^{2}(t)}\right] g\left(t, \mathbf{e}_{i}\right) \\
+\sum_{j \in E} q_{i j}\left[g\left(t, \mathbf{e}_{j}\right)-g\left(t, \mathbf{e}_{i}\right)\right]=0, \quad g(T, i)=1 .
\end{gathered}
$$

Remark 6. If the two risky assets are not correlated with each other, from Corollary 5, we can see that the optimal investment strategies are of the Merton solution types at a particular regime. Moreover, optimal investment strategies are directly proportional to the expected return and inversely proportional to the variance of the associated risky assets available to investors at such a regime. In this special case, we know each investor does not want to consider the other investor's strategy when he makes his optimal portfolio choice. It is equivalent to consider the classical utility maximization for individual's optimal portfolio choice under a regime switching financial market.

We note that the functions $f\left(t, \mathbf{e}_{i}\right)$ and $g\left(t, \mathbf{e}_{i}\right)$ in the expressions of the value functions given in Theorem 2 satisfy two systems of coupled linear ordinary differential equations. Generally speaking, differential equations are harder to solve. In what follows, we will derive another representation of $f\left(t, \mathbf{e}_{i}\right)$ and $g\left(t, \mathbf{e}_{i}\right)$ via the Feynman-Kac type representations which not only are more convenient to investigate the influence of the Markov-modulation on the value functions of the nonzero-sum stochastic differential portfolio game problem but also can better interpret and derive some properties of value functions. To derive the Feynman-Kac representations of both value functions $f\left(t, \mathbf{e}_{i}\right)$ and $g\left(t, \mathbf{e}_{i}\right)$, first we make the following assumptions of notation:

$$
\begin{aligned}
\mathbf{f}(t) & :=\left(f\left(t, \mathbf{e}_{1}\right), f\left(t, \mathbf{e}_{2}\right), \ldots, f\left(t, \mathbf{e}_{N}\right)\right)^{\prime}, \\
\mathbf{g}(t) & :=\left(g\left(t, \mathbf{e}_{1}\right), g\left(t, \mathbf{e}_{2}\right), \ldots, g\left(t, \mathbf{e}_{N}\right)\right)^{\prime},
\end{aligned}
$$

$$
\begin{aligned}
a_{i}:=a\left(t, \mathbf{e}_{i}\right) & \\
=\frac{\gamma_{1}^{2}}{2\left(1-\rho^{2}\right)}\left[\frac{\left(\mu_{2}(t)-r(t)\right)^{2}}{\gamma_{2}^{2} \sigma_{2}^{2}(t)}-\frac{\left(\mu_{1}(t)-r(t)\right)^{2}}{\gamma_{1}^{2} \sigma_{1}^{2}(t)}\right. & \\
& -2 \frac{\left(\mu_{2}(t)-r(t)\right)^{2}}{\gamma_{1} \gamma_{2} \sigma_{2}^{2}(t)} \\
& \left.+2 \rho \frac{\left(\mu_{1}(t)-r(t)\right)\left(\mu_{2}(t)-r(t)\right)}{\gamma_{1}^{2} \sigma_{1}(t) \sigma_{2}(t)}\right], \\
b_{i}:=b\left(t, \mathbf{e}_{i}\right) \quad & \frac{\left(\mu_{1}(t)-r(t)\right)^{2}}{\gamma_{1}^{2} \sigma_{1}^{2}(t)}-\frac{\left(\mu_{2}(t)-r(t)\right)^{2}}{\gamma_{2}^{2} \sigma_{2}^{2}(t)} \\
=\frac{\left(\mu_{1}(t)-r(t)\right)^{2}}{\gamma_{1} \gamma_{2} \sigma_{1}^{2}(t)} & \\
& \left.+2 \rho \frac{\left(\mu_{1}(t)-r(t)\right)\left(\mu_{2}(t)-r(t)\right)}{\gamma_{2}^{2} \sigma_{1}(t) \sigma_{2}(t)}\right] .
\end{aligned}
$$

We now give the Feynman-Kac representations of $f\left(t, \mathbf{e}_{i}\right)$ and $g\left(t, \mathbf{e}_{i}\right)$ in the following theorem.

Theorem 7. Let

$$
\begin{aligned}
& A(t):=\operatorname{diag}\left[a\left(t, \mathbf{e}_{1}\right), a\left(t, \mathbf{e}_{2}\right), \ldots, a\left(t, \mathbf{e}_{N}\right)\right]-Q, \\
& B(t):=\operatorname{diag}\left[b\left(t, \mathbf{e}_{1}\right), b\left(t, \mathbf{e}_{2}\right), \ldots, b\left(t, \mathbf{e}_{N}\right)\right]-Q,
\end{aligned}
$$

where diag $[x]$ denotes the diagonal matrix with diagonal elements given by the row vector $x$. Then the Feynman-Kac representations of $f\left(t, \mathbf{e}_{i}\right)$ and $g\left(t, \mathbf{e}_{i}\right)$ are given by

$$
\begin{aligned}
& f\left(t, \mathbf{e}_{i}\right)=E_{t, i}\left[\exp \left(\int_{t}^{T} a(u, \xi(u)) d u\right)\right], \\
& g\left(t, \mathbf{e}_{i}\right)=E_{t, i}\left[\exp \left(\int_{t}^{T} b(u, \xi(u)) d u\right)\right],
\end{aligned}
$$

where $a\left(t, \mathbf{e}_{i}\right)$ and $b\left(t, \mathbf{e}_{i}\right)$ are denoted by (41) and (42) and $E_{t, i}$ is the conditional expectation given that $\boldsymbol{\xi}(t)=\mathbf{e}_{i}$ under $\mathscr{P}$.

Proof. First, we will prove the Feynman-Kac representation of $f\left(t, \mathbf{e}_{i}\right)$. From Lemma 1.5 of Appendix B in [33], we know that the process $\{M(s) \mid s \in[t, T]\}$, defined by

$$
\begin{aligned}
M(s):= & f(s, \boldsymbol{\xi}(s))-f(t, \boldsymbol{\xi}(t)) \\
& -\int_{t}^{s}\left(f_{t}(u, \xi(u))+\left\langle\mathbf{f}(u), Q^{\prime} \boldsymbol{\xi}(u)\right\rangle\right) d u,
\end{aligned}
$$

is an $\left(\mathscr{F}^{\xi}, \mathscr{P}\right)$-martingale. Next, we define a function $R(s)$ by

$$
R(s):=\exp \left(\int_{t}^{s} a(u, \xi(u)) d u\right) .
$$


Then by applying the product rule of Ito formula and the definition of $M(s)$, we obtain

$$
R(s) f(s, \xi(s))-f(t, \xi(t))=\int_{t}^{s} R(u) d M(u) .
$$

Setting $s=T$ and taking expectation, we obtain

$$
f\left(t, \mathbf{e}_{i}\right)=E_{t, i}[R(T)]=E_{t, i}\left[\exp \left(\int_{t}^{T} a(u, \xi(u)) d u\right)\right] .
$$

We note that the proof of the Feynman-Kac representation of function $g\left(t, \mathbf{e}_{i}\right)$ is the same as the above process, so we omit it here.

From the expressions of $f\left(t, \mathbf{e}_{i}\right)$ in (44) and $g\left(t, \mathbf{e}_{i}\right)$ in (45), it is not difficult to see $f\left(t, \mathbf{e}_{i}\right)>0$ and $g\left(t, \mathbf{e}_{i}\right)>0$. Consequently, for each $k=1,2, V_{k}\left(t, z, \mathbf{e}_{i}\right)$ given by (33) indeed satisfy $V_{k, z}^{i}>0$ and $V_{k, z z}^{i}<0$ just as described in the previous section.

Remark 8. By using the notations of $(A(t)$ or $B(t))$ and $(\mathbf{f}(t)$ or $\mathbf{g}(t))$, the matrix form of (31) and (32) can be denoted by

$$
\begin{aligned}
\mathbf{f}^{\prime}(t)=A(t) \mathbf{f}(t), & \mathbf{f}(T)=\mathbf{1}, \\
\mathbf{g}^{\prime}(t)=B(t) \mathbf{g}(t), & \mathbf{g}(T)=\mathbf{1},
\end{aligned}
$$

where $\mathbf{1}=(1,1, \ldots, 1)^{\prime} \in \mathfrak{R}^{N}$. The differential equation systems (50) indeed have unique continuous solutions referred to in (page 303, Theorem 1) of [40]. For more similar discussions on the existence of solutions of the differential equation system, interested readers can refer to Remark 4.2 of [41].

\section{One Special Case}

In order to investigate how the Markov regime switching influences optimal investment strategies and value functions and provide meaningful comparative statics analysis, in this section we present one special case of the nonzero-sum stochastic differential portfolio game problem established in Sections 3 and 4 . We will assume that the interest rate denoted by $r(t, \xi(t)) \equiv r, t \in \mathscr{T}$ is not modulated by Markov chain. Namely, the price of bank account is not affected by the external conditions. This assumption is relatively reasonable since compared to risky assets, the price of the bank account is more stable. Furthermore, we assume $\mathcal{S}:=\{1,2\}$; that is, $\boldsymbol{\xi}:=\{\boldsymbol{\xi}(t) \mid t \in \mathscr{T}\}$ is a two-state Markov chain. We first derive regime switching HJB equations for value functions of this simplified model. We assume that the rate matrix $Q$ of the Markov chain is given by

$$
Q=\left(\begin{array}{cc}
-q & q \\
q & -q
\end{array}\right)
$$

where $q$ is a positive real constant. Note that it is not necessary to consider the Markov chain with only two states. It just increases computation complexity in the numerical analysis section given a general rate matrix $Q$. Furthermore, we know that the two-state Markov chain is rich enough to distinguish a "bull" market and a "bear" market. In this case, from Theorem 2, we see that the value functions of investors A and $\mathrm{B}$ for the two-state economy are denoted by the following forms:

$$
\begin{array}{ll}
V_{1}^{i}=V_{1}(t, z, i)=-\frac{1}{\gamma_{1}} e^{-\gamma_{1} z e^{r(T-t)}} f(t, i), & i=1,2, \\
V_{2}^{i}=V_{2}(t, z, i)=-\frac{1}{\gamma_{2}} e^{-\gamma_{2} z e^{r(T-t)}} g(t, i), & i=1,2,
\end{array}
$$

where $f(t, i)$ is the solutions of the following pair of linear ordinary differential equations:

$$
\begin{aligned}
& \frac{\partial f(t, 1)}{\partial t}+\frac{\gamma_{1}^{2}}{2\left(1-\rho^{2}\right)}\left[\frac{\left(\mu_{2}^{1}-r\right)^{2}}{\gamma_{2}^{2}\left(\sigma_{2}^{1}\right)^{2}}-\frac{\left(\mu_{1}^{1}-r\right)^{2}}{\gamma_{1}^{2}\left(\sigma_{1}^{1}\right)^{2}}-2 \frac{\left(\mu_{2}^{1}-r\right)^{2}}{\gamma_{1} \gamma_{2}\left(\sigma_{2}^{1}\right)^{2}}\right. \\
& \left.+2 \rho \frac{\left(\mu_{1}^{1}-r\right)\left(\mu_{2}^{1}-r\right)}{\gamma_{1}^{2} \sigma_{1}^{1} \sigma_{2}^{1}}\right] f(t, 1) \\
& +q[f(t, 2)-f(t, 1)]=0, \\
& \frac{\partial f(t, 2)}{\partial t}+\frac{\gamma_{1}^{2}}{2\left(1-\rho^{2}\right)}\left[\frac{\left(\mu_{2}^{2}-r\right)^{2}}{\gamma_{2}^{2}\left(\sigma_{2}^{2}\right)^{2}}-\frac{\left(\mu_{1}^{2}-r\right)^{2}}{\gamma_{1}^{2}\left(\sigma_{1}^{2}\right)^{2}}-2 \frac{\left(\mu_{2}^{2}-r\right)^{2}}{\gamma_{1} \gamma_{2}\left(\sigma_{2}^{2}\right)^{2}}\right. \\
& \left.+2 \rho \frac{\left(\mu_{1}^{2}-r\right)\left(\mu_{2}^{2}-r\right)}{\gamma_{1}^{2} \sigma_{1}^{2} \sigma_{2}^{2}}\right] f(t, 2) \\
& -q[f(t, 2)-f(t, 1)]=0,
\end{aligned}
$$

with boundary conditions $f(T, 1)=1$ and $f(T, 2)=1$, and $g(t, i)$ is the solutions of the following pair of linear ordinary differential equations:

$$
\begin{gathered}
\frac{\partial g(t, 1)}{\partial t}+\frac{\gamma_{2}^{2}}{2\left(1-\rho^{2}\right)}\left[\frac{\left(\mu_{1}^{1}-r\right)^{2}}{\gamma_{1}^{2}\left(\sigma_{1}^{1}\right)^{2}}-\frac{\left(\mu_{2}^{1}-r\right)^{2}}{\gamma_{2}^{2}\left(\sigma_{2}^{1}\right)^{2}}-2 \frac{\left(\mu_{1}^{1}-r\right)^{2}}{\gamma_{1} \gamma_{2}\left(\sigma_{1}^{1}\right)^{2}}\right. \\
\left.+2 \rho \frac{\left(\mu_{1}^{1}-r\right)\left(\mu_{2}^{1}-r\right)}{\gamma_{2}^{2} \sigma_{1}^{1} \sigma_{2}^{1}}\right] g(t, 1) \\
+q[g(t, 2)-g(t, 1)]=0,
\end{gathered}
$$




$$
\begin{gathered}
\frac{\partial g(t, 2)}{\partial t}+\frac{\gamma_{2}^{2}}{2\left(1-\rho^{2}\right)}\left[\frac{\left(\mu_{1}^{2}-r\right)^{2}}{\gamma_{1}^{2}\left(\sigma_{1}^{2}\right)^{2}}-\frac{\left(\mu_{2}^{2}-r\right)^{2}}{\gamma_{2}^{2}\left(\sigma_{2}^{2}\right)^{2}}-2 \frac{\left(\mu_{1}^{2}-r\right)^{2}}{\gamma_{1} \gamma_{2}\left(\sigma_{1}^{2}\right)^{2}}\right. \\
\left.+2 \rho \frac{\left(\mu_{1}^{2}-r\right)\left(\mu_{2}^{2}-r\right)}{\gamma_{2}^{2} \sigma_{1}^{2} \sigma_{2}^{2}}\right] g(t, 2) \\
-q[g(t, 2)-g(t, 1)]=0,
\end{gathered}
$$

with boundary conditions $g(T, 1)=1$ and $g(T, 2)=1$.

For the sake of conciseness, we can rewrite (53)-(54) in the following simplified forms by using the notations of $a_{i}$ in (41) and $b_{i}$ in (42):

$$
\begin{aligned}
& \frac{\partial f(t, 1)}{\partial t}+\left(a_{1}-q\right) f(t, 1)+q f(t, 2)=0, \quad f(T, 1)=1, \\
& \frac{\partial f(t, 2)}{\partial t}+\left(a_{2}-q\right) f(t, 2)+q f(t, 1)=0, \quad f(T, 2)=1,
\end{aligned}
$$

$$
\begin{array}{ll}
\frac{\partial g(t, 1)}{\partial t}+\left(b_{1}-q\right) g(t, 1)+q g(t, 2)=0, & g(T, 1)=1, \\
\frac{\partial g(t, 2)}{\partial t}+\left(b_{2}-q\right) g(t, 2)+q g(t, 1)=0, & g(T, 2)=1
\end{array}
$$

and the optimal portfolio strategies of investors A and B for this case are, respectively, given by

$$
\begin{aligned}
& \pi_{1}^{*}(t, 1)=\frac{1}{1-\rho^{2}}\left(\frac{\mu_{1}^{1}-r}{\gamma_{1}\left(\sigma_{1}^{1}\right)^{2}} e^{-r(T-t)}-\rho \frac{\mu_{2}^{1}-r}{\gamma_{2} \sigma_{1}^{1} \sigma_{2}^{1}} e^{-r(T-t)}\right), \\
& \pi_{1}^{*}(t, 2)=\frac{1}{1-\rho^{2}}\left(\frac{\mu_{1}^{2}-r}{\gamma_{1}\left(\sigma_{1}^{2}\right)^{2}} e^{-r(T-t)}-\rho \frac{\mu_{2}^{2}-r}{\gamma_{2} \sigma_{1}^{2} \sigma_{2}^{2}} e^{-r(T-t)}\right), \\
& \pi_{2}^{*}(t, 1)=\frac{1}{1-\rho^{2}}\left(\frac{\mu_{2}^{1}-r}{\gamma_{2}\left(\sigma_{2}^{1}\right)^{2}} e^{-r(T-t)}-\rho \frac{\mu_{1}^{1}-r}{\gamma_{1} \sigma_{1}^{1} \sigma_{2}^{1}} e^{-r(T-t)}\right), \\
& \pi_{2}^{*}(t, 2)=\frac{1}{1-\rho^{2}}\left(\frac{\mu_{2}^{2}-r}{\gamma_{2}\left(\sigma_{2}^{2}\right)^{2}} e^{-r(T-t)}-\rho \frac{\mu_{1}^{2}-r}{\gamma_{1} \sigma_{1}^{2} \sigma_{2}^{2}} e^{-r(T-t)}\right) .
\end{aligned}
$$

We know that the above two pairs of linear differential equations for functions $f$ and $g$ (see (55) and (56)) can be explicitly solved. To solve these two differential equations, we first give the following lemma.

Lemma 9. Let $r_{1,2}$ and $r_{3,4}$ be the solutions of the following two quadratic equations, respectively:

$$
\begin{aligned}
& r^{2}+\left(a_{1}+a_{2}-2 q\right) r+\left[\left(a_{1}-q\right)\left(a_{2}-q\right)-q^{2}\right]=0, \\
& r^{2}+\left(b_{1}+b_{2}-2 q\right) r+\left[\left(b_{1}-q\right)\left(b_{2}-q\right)-q^{2}\right]=0 .
\end{aligned}
$$

Then one can easily obtain

$$
\begin{aligned}
& r_{1,2}=\frac{-\left(a_{1}+a_{2}-2 q\right) \pm \sqrt{\left(a_{1}-a_{2}\right)^{2}+4 q^{2}}}{2}, \\
& r_{3,4}=\frac{-\left(b_{1}+b_{2}-2 q\right) \pm \sqrt{\left(b_{1}-b_{2}\right)^{2}+4 q^{2}}}{2},
\end{aligned}
$$

where the notations $a_{i}, b_{i}$, and $q$ are given by (41), (42), and (51).

Proof. The conclusion of this lemma follows directly from the solutions of quadratic equation. Meanwhile, the discriminants of the quadratic equations are always bigger than zero, so the equations have two different real roots.

For mathematical convenience, let us denote

$$
\begin{aligned}
& C_{1}=\frac{\left(r_{2}+a_{2}\right)\left(r_{1}+a_{2}-q\right)}{q\left(r_{1}-r_{2}\right)}, \\
& C_{2}=-\frac{\left(r_{1}+a_{2}\right)\left(r_{2}+a_{2}-q\right)}{q\left(r_{1}-r_{2}\right)}, \\
& C_{3}=-\frac{\left(r_{2}+a_{2}\right)}{\left(r_{1}-r_{2}\right)}, \quad C_{4}=\frac{\left(r_{1}+a_{2}\right)}{\left(r_{1}-r_{2}\right)}, \\
& C_{5}=\frac{\left(r_{4}+b_{2}\right)\left(r_{3}+b_{2}-q\right)}{q\left(r_{3}-r_{4}\right)}, \\
& C_{6}=-\frac{\left(r_{3}+b_{2}\right)\left(r_{4}+b_{2}-q\right)}{q\left(r_{3}-r_{4}\right)}, \\
& C_{7}=-\frac{\left(r_{4}+b_{2}\right)}{\left(r_{3}-r_{4}\right)}, \quad C_{8}=\frac{\left(r_{3}+b_{2}\right)}{\left(r_{3}-r_{4}\right)} .
\end{aligned}
$$

Then the solutions of $f(t, 1), f(t, 2)$ and $g(t, 1), g(t, 2)$ are explicitly given by

$$
\begin{aligned}
& f(t, 1)=C_{1} e^{-r_{1}(T-t)}+C_{2} e^{-r_{2}(T-t),}, \\
& f(t, 2)=C_{3} e^{-r_{1}(T-t)}+C_{4} e^{-r_{2}(T-t)}, \\
& g(t, 1)=C_{5} e^{-r_{3}(T-t)}+C_{6} e^{-r_{4}(T-t)}, \\
& g(t, 2)=C_{7} e^{-r_{3}(T-t)}+C_{8} e^{-r_{4}(T-t)} .
\end{aligned}
$$

Remark 10. When the model parameters $\mu_{k}$ and $\sigma_{k}$ of risky assets for each $k=1,2$ in the two regimes are identical to each other, respectively, this result will lead to $a_{1}=a_{2}:=a$ and $b_{1}=b_{2}:=b$. No matter what the value of the parameter $q$ in the rate matrix of the Markov chain is, we find that the optimal value functions and optimal portfolio strategies are robust with respect to the change in the value of $q$. In this case, the above two pairs of linear ordinary differential equations (53) or (55) and (54) or (56) with the corresponding boundary conditions have unique solutions, respectively. In this case, we can easily obtain the solutions of $f$ and $g$. That is,

$$
f(t)=e^{a(T-t)} \quad g(t)=e^{b(T-t)},
$$


where this result is just as described in Remark 4 in Section 4. This case is equivalent to the assumption that Markov chain has only one state.

\section{Comparative Statics and Numerical Simulation}

To gain more insight into the economic significance of regime switching on the optimal portfolio strategies, in this section we will construct numerical analysis to investigate how optimal investment strategies change with the parameters arising from our Markov-modulated model, for example, the absolute risk aversion coefficients of two investors and the correlation coefficient between the two risky assets. We also make comparisons of the qualitative behaviors of the optimal portfolio strategies obtained from our model (Model I) to those arising from the model without regimes (Model II).

To perform both the comparative statics analysis and the comparison between Model I and Model II simultaneously, we implement the procedure just as [14]. For illustration, we consider the special case described in Section 5 where Markov chain is assumed to only have two states and interest rate is fixed. Here we also suppose that the two states in Markov chain $\boldsymbol{\xi}$ represent economy 1 (E1) and economy 2 (E2), respectively.

In order to make the comparison effectively, our numerical results are based on the following annualized baseline hypothetical values for the model parameters unless otherwise stated: the absolute risk aversion coefficients $\gamma_{1}=0.4$, $\gamma_{2}=0.2$, the current time $t=6$, the mature horizon $T=10$, the risk-free interest rate $r=0.05$, the drift rates of the two risky assets $\mu_{1}^{1}=0.08, \mu_{2}^{1}=0.1$, and the volatility rates of the two risky assets $\sigma_{1}^{1}=0.3, \sigma_{2}^{1}=0.5$. These hypothetical parameter values are drawn from those used in [29], because we simultaneously want to make comparisons of the parameter sensitivity analysis results obtained from our model to the properties obtained in [29].

For each $k=1,2$, when the drift rates $\mu_{k}^{2}$ and the volatility rates $\sigma_{k}^{2}$ of the risky assets in E2 are the same as their corresponding parameter values in E1, we say that Model I and Model II are identical to each other. The numerical results in this case for the optimal portfolio strategies obtained from Model I are identical to those arising from Model II whatever the value of the parameter $q$ in the rate matrix of Markov chain $Q$ is. Thus the results are robust with respect to the change of $q$. Interested readers can refer to Remark 10 for details about this description.

In the next, we implement the numerical analysis for the optimal investment strategies with respect to that particular parameter. We will focus on how the optimal portfolio strategies vary against model parameters when the economy state modeled by Markov chain changes, namely, how the regime shifts in market parameters $\mu_{k}^{2}$ and $\sigma_{k}^{2}$ for each $k=1,2$ affect portfolio strategies. In the following two subsections, we analyze this issue along two dimensions. Firstly, we investigate the impact of regime shifts in drift rates $\mu_{k}^{2}, k=1,2$, on optimal portfolio strategies against the absolute risk aversion coefficients and correlation coefficient, respectively. Secondly,

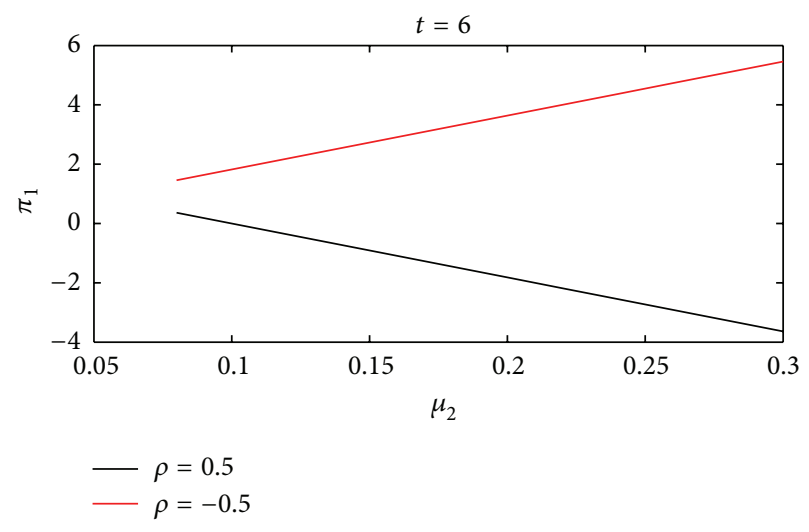

FIgURE 1: Optimal strategy $\pi_{1}$ against $\mu_{2}$ for different $\rho$.

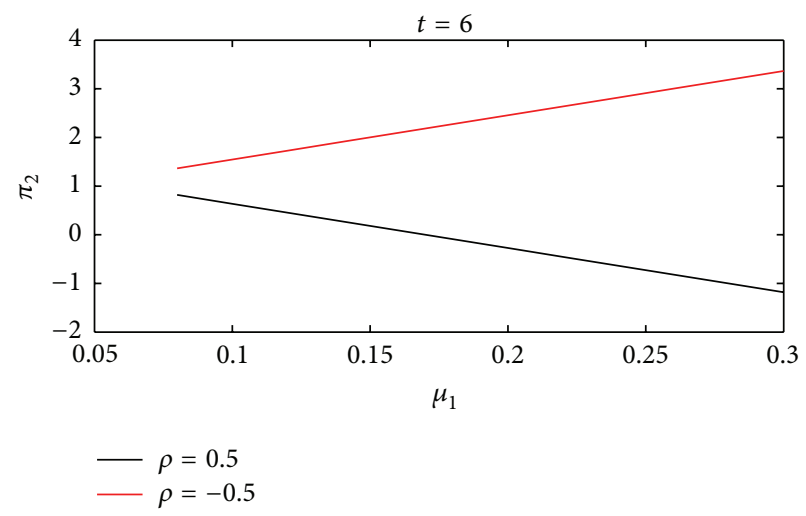

FIgURE 2: Optimal strategy $\pi_{2}$ against $\mu_{1}$ for different $\rho$.

we analyze the effects of regime switching in volatilities of financial assets on the portfolio strategies against the same parameters as those with respect to the analysis of the impact of regime shifts in drift rates.

6.1. The Effect of Drift Rates. The main purpose of this subsection is to see the effects of the drift rates $\mu_{1}^{2}$ and $\mu_{2}^{2}$ of the two risky assets in economy E2 on the optimal portfolio strategies. We suppose there are three cases for our model parameters, namely, $\mu_{1}^{2}>\mu_{1}^{1}$ or $\mu_{2}^{2}>\mu_{2}^{1}$ or the two conditions $\mu_{1}^{2}>\mu_{1}^{1}$ and $\mu_{2}^{2}>\mu_{2}^{1}$ satisfying simultaneously. When only one of the three conditions satisfies, then economy E1 is said to be a "good" economy relative to economy E2. Or else E1 is a "bad" economy. In this case, Model I and Model II are said to be different from each other. However, for each $k=1,2$, when $\mu_{k}^{1}=\mu_{k}^{2}$ (i.e., E1 and E2 coincide), it means that the two states are identical. It is equivalent to say the model has no regime switching and Model I and Model II are identical to each other.

Figures 1 and 2 plot the current optimal strategies $\pi_{1}$ against the drift rate $\mu_{2}$ and $\pi_{2}$ against the drift rate $\mu_{1}$ for two different, opposite sign, particular values of correlation coefficient $\rho$, respectively. Both figures show the fundamental properties of optimal portfolio strategies in a benchmark model in which there are no regimes in the financial markets. 


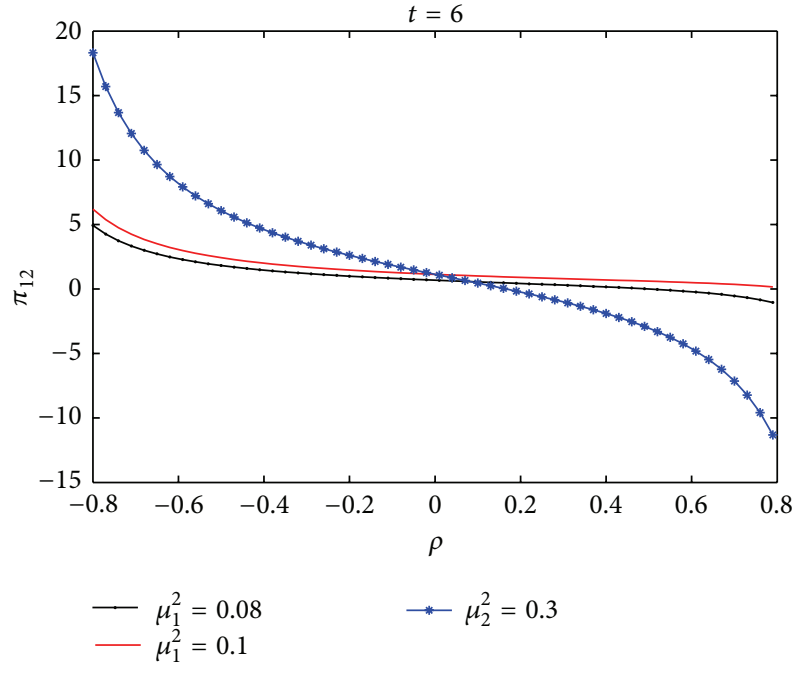

FIGURE 3: Optimal $\pi_{1}$ in E2 against $\rho$ for different $\mu_{k}^{2}$.

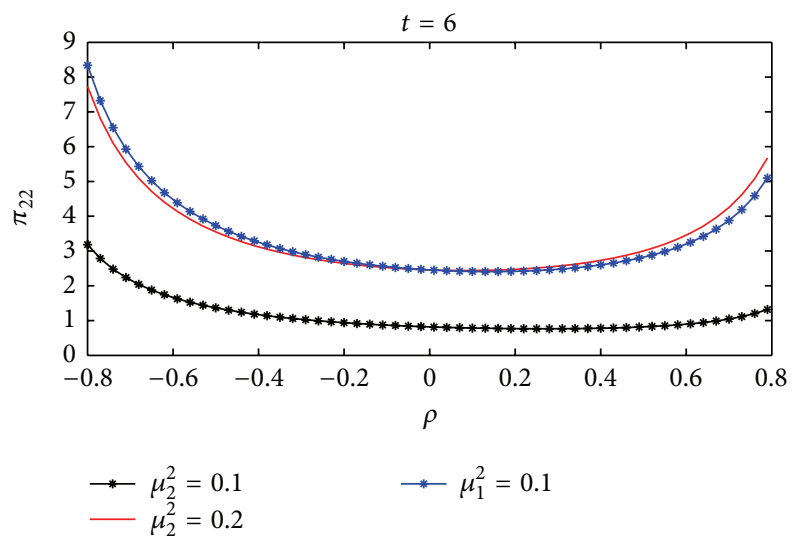

FIGURE 4: Optimal $\pi_{2}$ in E2 against $\rho$ for different $\mu_{k}^{2}$.

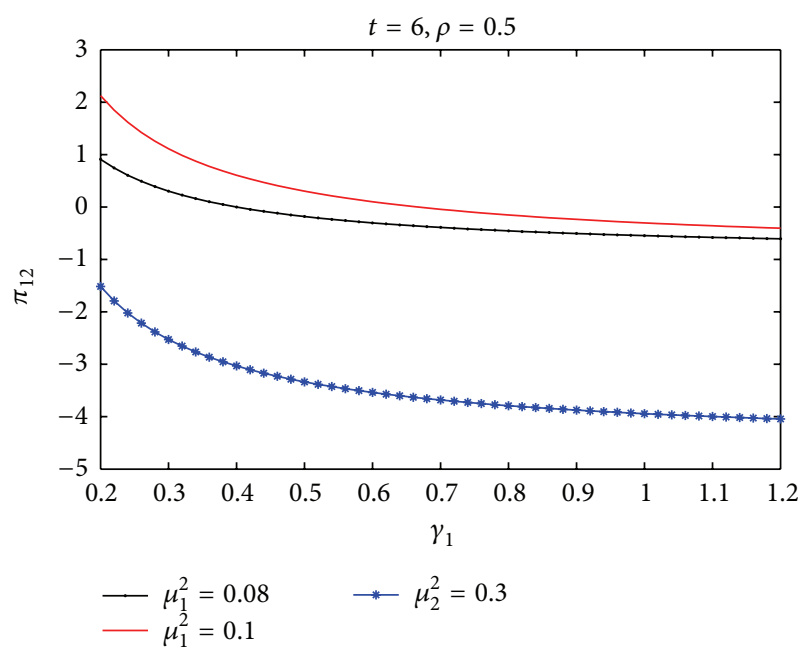

FIGURE 5: $\pi_{1}$ in E2 against $\gamma_{1}$ for $\rho=0.5$ and different $\mu_{k}^{2}$.

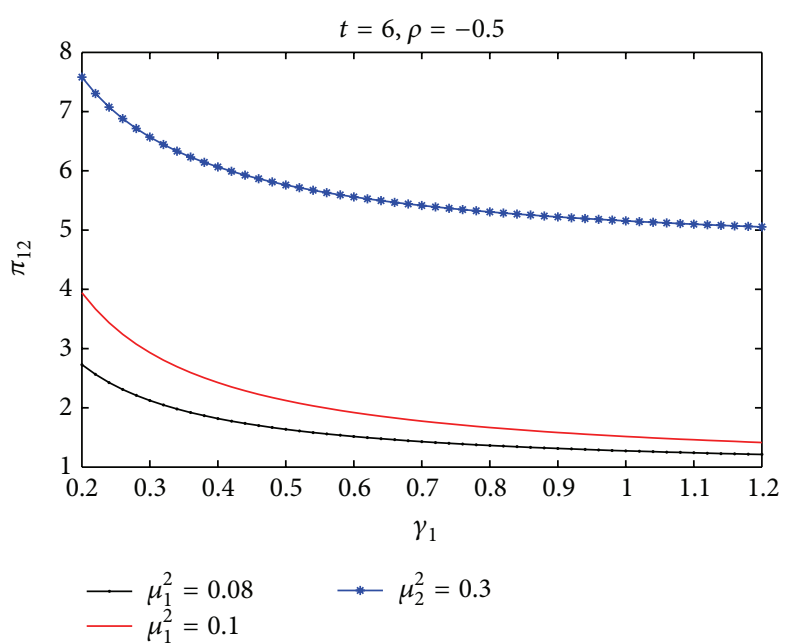

FIgURE 6: $\pi_{1}$ in E2 against $\gamma_{1}$ for $\rho=-0.5$ and different $\mu_{k}^{2}$.

From Figure 1, it can be easily seen that $\pi_{1}$ decreases as $\mu_{2}$ increases when $\rho>0$, while it increases when $\rho<0$. This property of portfolio strategy $\pi_{1}$ similarly applies to $\pi_{2}$.

Figures 3 and 4 depict the plots of the optimal investment strategies $\pi_{1}$ and $\pi_{2}$ against the correlation coefficient $\rho$ for different values of $\mu_{k}^{2}$, respectively. The plots compare the two cases when there is no regime $\left(\mu_{2}^{2}=\mu_{2}^{1}=0.1, \mu_{1}^{2}=\right.$ $\left.\mu_{1}^{1}=0.08\right)$ and when the state changes to E2. We can see that the regime switching in drift rates has a significant impact on the qualitative behavior of optimal portfolio strategies. According to (57) and (58), for each $k=1,2$, we can easily know that $\pi_{k}$ increases with $\mu_{k}$. From Figure 3 , we can see that $\pi_{1}$ increases along with $\mu_{1}^{2}$. When $\rho<0$, it substantially increases as $\mu_{2}^{2}$. However, it significantly decreases as $\mu_{2}^{2}$ increases when $\rho>0$. According to Theorem 5.1 in [29], the optimal portfolio strategy $\pi_{1}$ decreases with $\rho$ when $\theta_{1} / \gamma_{1}<$ $\theta_{2} / \gamma_{1}$. Here the parameters given in Model I and Model II satisfy this condition. Likewise, from Figure 4, we see that the optimal investment $\pi_{2}$ substantially increases along with $\mu_{2}^{2}$ and also increases as $\mu_{1}^{2}$ when $\rho<0$. While $\rho>0, \pi_{2}$ slightly decreases as $\mu_{1}^{2}$ increases. Moreover, for the three cases when $\mu_{2}^{2}=0.1, \mu_{2}^{2}=0.2$, and $\mu_{1}^{2}=0.1$, there is a critical point at which there is a reversal of the behavior of optimal investment strategy from decreasing to increasing against different values of $\rho$.

Figures 5 and 6 depict the plots of optimal investment strategy $\pi_{1}$ against the absolute risk aversion coefficient $\gamma_{1}$. Similar to the above, in these figures we compare the two cases when there is no regime $\left(\mu_{2}^{2}=\mu_{2}^{1}=0.1, \mu_{1}^{2}=\mu_{1}^{1}=0.08\right)$ and when the state changes to E2. From Figures 5 and 6, for all the three cases when $\mu_{1}^{1}=0.08, \mu_{1}^{2}=0.1$, and $\mu_{2}^{2}=0.3$, we see that the optimal portfolio strategy $\pi_{1}$ decreases as risk aversion coefficient $\gamma_{1}$ increases. Also, $\pi_{1}$ increases along with $\mu_{1}^{2}$ whenever the values of correlation coefficient $\rho$ take. At the same time, Figure 5 shows that optimal strategy $\pi_{1}$ significantly decreases as $\mu_{2}^{2}$ increases when $\rho>0$. However, we find that this result is opposite when $\rho<0$ in Figure 6. 


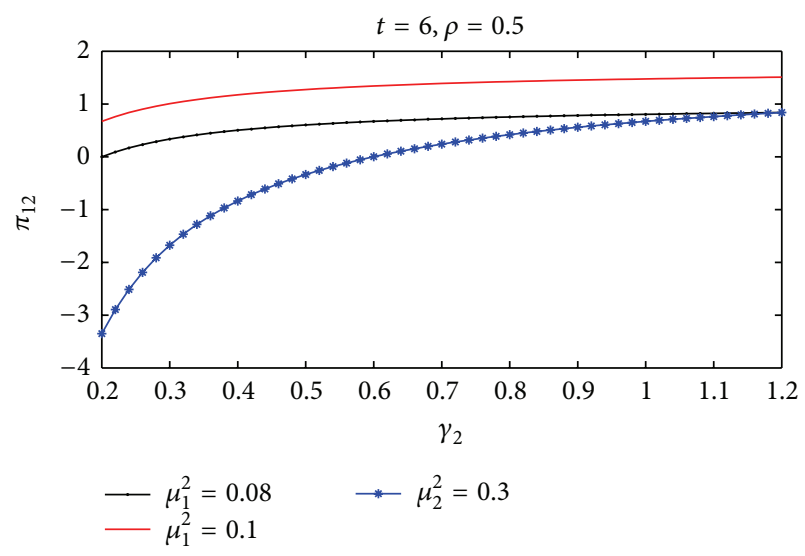

FIGURE 7: $\pi_{1}$ in E2 against $\gamma_{2}$ for $\rho=0.5$ and different $\mu_{k}^{2}$.

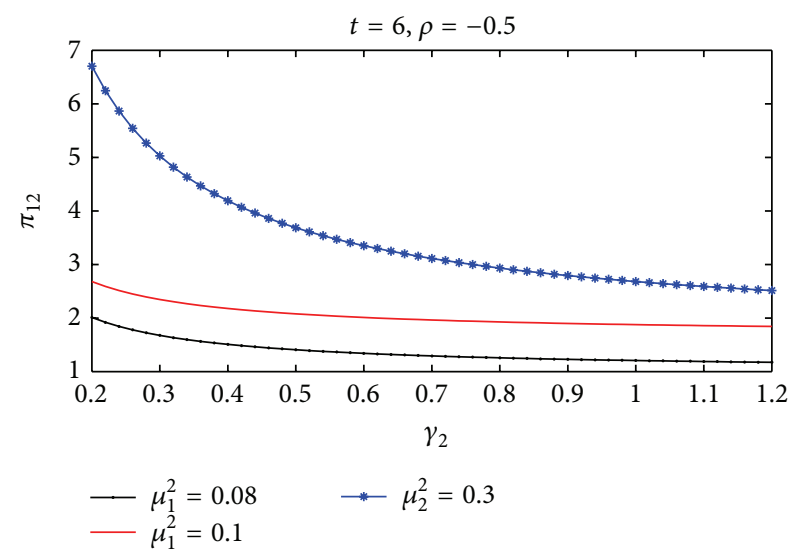

Figure 8: $\pi_{1}$ in E2 against $\gamma_{2}$ for $\rho=-0.5$ and different $\mu_{k}^{2}$.

Figures 7 and 8 plot $\pi_{1}$ against the absolute risk aversion coefficient $\gamma_{2}$. From Figures 7 and 8 , we see that the optimal investment strategy increases as $\mu_{1}^{2}$ increases and also substantially decreases as $\mu_{2}^{2}$ increases when $\rho>0$. While $\rho<0$, it shows the opposite result. The optimal investment significantly increases along with $\mu_{2}^{2}$. For all the three cases when $\mu_{2}^{1}=0.1, \mu_{2}^{2}=0.2$, and $\mu_{1}^{2}=0.1, \pi_{1}$ increases with $\gamma_{2}$ when $\rho>0$. When $\rho<0$, it decreases with $\gamma_{2}$. This result is consistent with Theorem 5.2 in [29].

Figures 9 and 10 and Figures 11 and 12 describe the effects of $\mu_{k}^{2}$ on the qualitative behavior of the optimal portfolio strategy $\pi_{2}$ of investor B against the risk aversion coefficients $\gamma_{1}$ and $\gamma_{2}$ for different values of $\rho$. Since the explanations for these figures are similar to the above analysis of optimal portfolio strategy of investor A, we omit them here. From the above figures, we can see that regime switching represented by the switches of drift rate $\mu$ really has a significant impact on the optimal investment strategy. To consider regime switching models will help to incorporate the structure change of the model and help investors to adjust their investment strategies according to the changing conditions of the macroeconomy.

6.2. The Effect of Volatility Rates. In this subsection we will focus on the effect of the volatility rates $\sigma_{1}^{2}$ and $\sigma_{2}^{2}$ of the risky

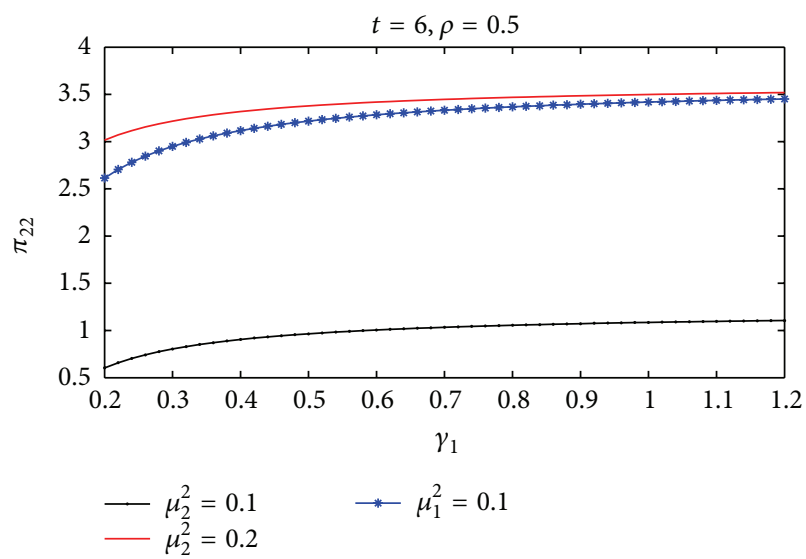

FIGURE 9: $\pi_{2}$ in E2 against $\gamma_{1}$ for $\rho=0.5$ and different $\mu_{k}^{2}$.

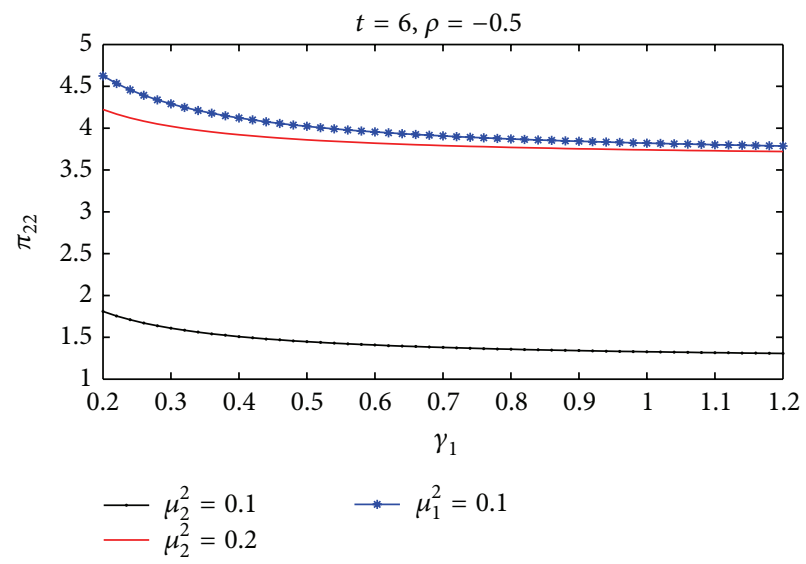

FIGURE 10: $\pi_{2}$ in E2 against $\gamma_{1}$ for $\rho=-0.5$ and different $\mu_{k}^{2}$.

assets in E2 on the optimal portfolio strategies. We suppose there are three cases for our model parameters, namely, $\sigma_{1}^{2}>$ $\sigma_{1}^{1}$ or $\sigma_{2}^{2}>\sigma_{2}^{1}$ or the two conditions $\sigma_{1}^{2}>\sigma_{1}^{1}$ and $\sigma_{2}^{2}>\sigma_{2}^{1}$ satisfying simultaneously. When one of the three conditions satisfies, then economy E1 is said to be a "bad" economy relative to E2. Or else E1 is a "good" economy. In this case Model I and Model II are different from each other. For each $k=1,2$, when $\sigma_{k}^{1}=\sigma_{k}^{2}$ (i.e., E1 and E2 coincide), it means that the model has no regime and we say Model I and Model II are identical. From the following comparative static analysis, we can see that the effects of regime switching on the optimal investment strategies are significant.

Figures 13 and 14 describe the plots of optimal investment strategies $\pi_{1}$ and $\pi_{2}$ against the correlation coefficient for different values of $\sigma_{k}^{2}$. The plots compare when there is no regime $\left(\sigma_{2}^{2}=\sigma_{2}^{1}, \sigma_{1}^{2}=\sigma_{1}^{1}\right)$ and when the state changes to E2. From Figure 13, we see that the optimal investment $\pi_{1}$ decreases when $\sigma_{2}^{2}$ increases for $\rho<0$. This result is opposite for $\rho>0$. From Figure 14, it shows that the optimal investment $\pi_{2}$ decreases when $\sigma_{1}^{2}$ increases for $\rho<0$. However, for $\rho>0$, it is an increasing function of $\sigma_{1}^{2}$. This relation for both investment strategies at a particular regime is consistent with Theorem 5.3 in [29]. 


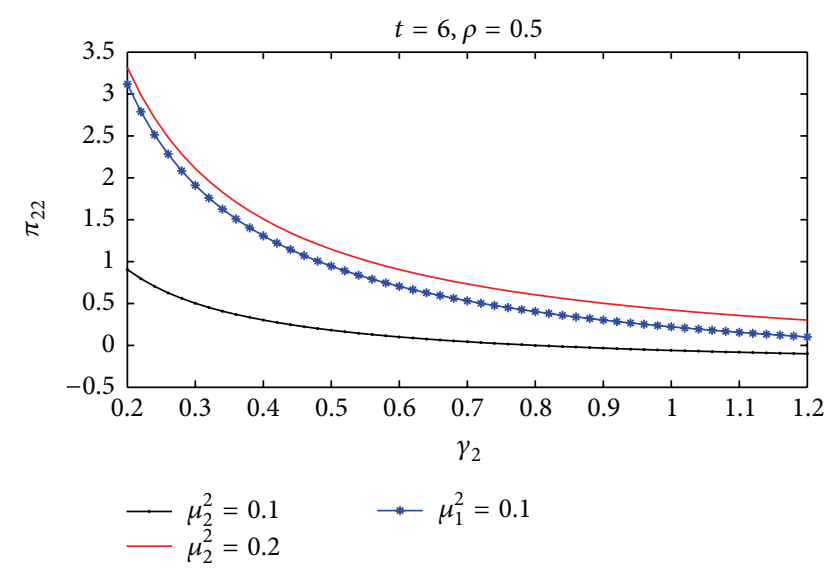

FIGURE 11: $\pi_{2}$ in E2 against $\gamma_{2}$ for $\rho=0.5$ and different $\mu_{k}^{2}$.

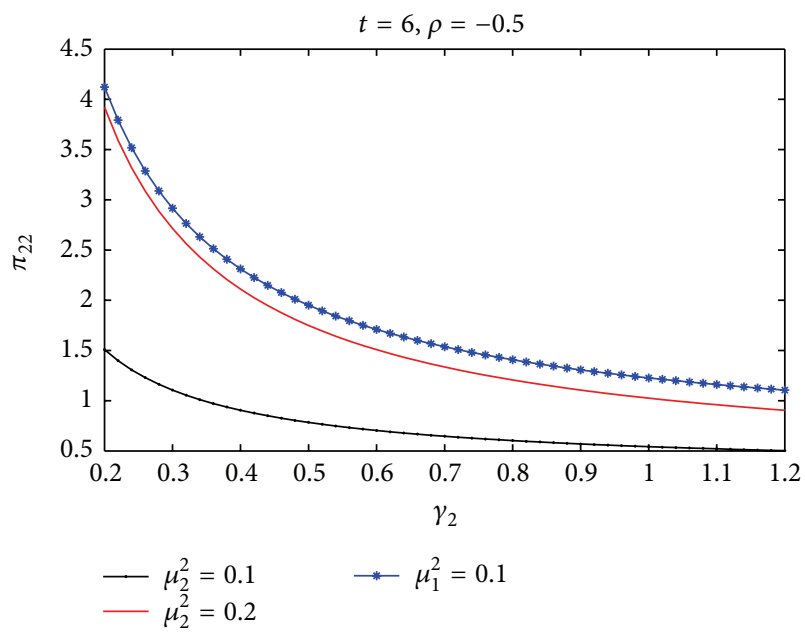

FIGURE 12: $\pi_{2}$ in E2 against $\gamma_{2}$ for $\rho=-0.5$ and different $\mu_{k}^{2}$.

And the monotonicity properties between two investment strategies and correlation coefficient for regime shifts in volatilities are the same as the analysis of drift rates in Figures 3 and 4 .

Figures 15, 16, 17, and 18 depict the plots of the optimal portfolio strategy $\pi_{1}$ against the risk aversion coefficients $\gamma_{1}$ and $\gamma_{2}$ for different values of $\rho$ when the regime switching is represented by switches of volatilities $\sigma_{k}^{2}$. The results showed in all these figures reflect that the regime switching in volatility has a significant impact on the optimal portfolio strategy. The monotonicity properties between investment and risk aversion coefficient $\gamma_{1}$ are the same for different values of $\rho$ as what are described in detail in the above subsections for the effect of drift rates. From Figure 15, when $\rho$ takes the positive value and the volatility $\sigma_{1}^{2}$ increases from 0.3 to 0.5 , we can see there is a critical point at which there is a reversal of the optimal investment strategy from less to more. However, in Figure 16, when $\rho=-0.5$ the optimal investment strategy in no regime $\left(\sigma_{1}^{2}=0.3\right)$ is more than the case when there is regime. Based on the case for $\sigma_{1}^{2}=0.5$, Figure 15

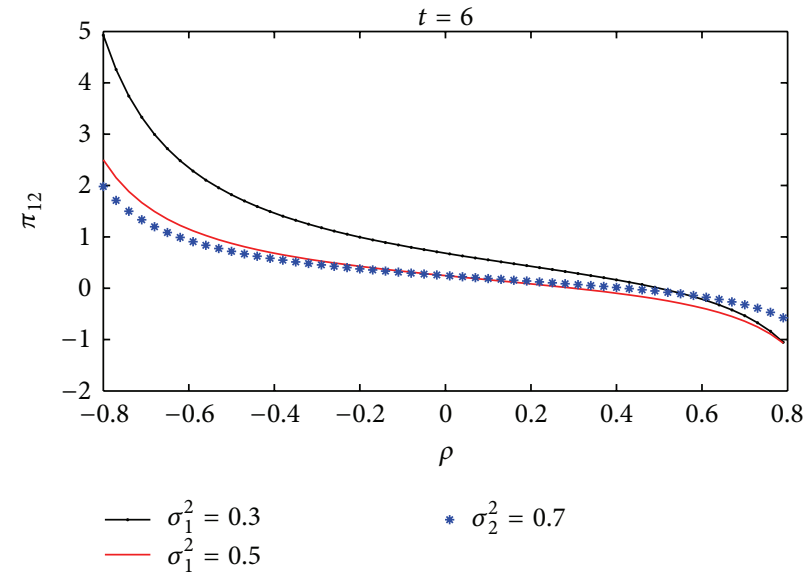

FIGURE 13: $\pi_{1}$ in E2 against $\rho$ for different $\sigma_{k}^{2}$.

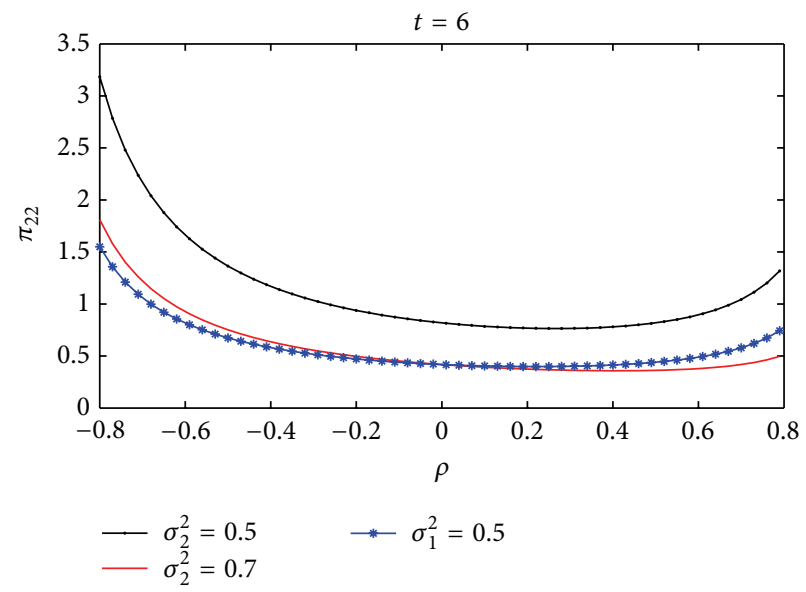

FIGURE 14: $\pi_{2}$ in E2 against $\rho$ for different $\sigma_{k}^{2}$.

shows that the optimal investment strategy becomes bigger when $\rho=0.5$; however, this result is opposite in Figure 16.

In Figures 17 and 18, we can see the monotonicity properties of the optimal investment strategy $\pi_{1}$ against the risk aversion $\gamma_{2}$ are the exact opposite when $\rho$ takes arbitrary positive or negative value. And also compared to the no regime switching case when $\sigma_{1}^{2}=0.3$, the optimal investment strategy $\pi_{1}$ in the two regime switching cases $\left(\sigma_{1}^{2}=0.5\right.$ or $\sigma_{1}^{2}=0.5$ and $\left.\sigma_{2}^{2}=0.7\right)$ decreases significantly.

Figures 19, 20, 21, and 22 plot the optimal investment strategy $\pi_{2}$ against the risk aversion coefficients $\gamma_{1}$ and $\gamma_{2}$ for different values of $\rho$ when the regime switching is represented by switches of $\sigma_{k}^{2}$. In Figures 19 and 20, we can see the monotonicity relations between optimal investment strategies $\pi_{2}$ and $\gamma_{1}$ are exactly opposite when $\rho$ takes positive or negative value, while in Figures 21 and 22, the optimal investment strategy $\pi_{2}$ decreases as $\gamma_{2}$ increases for different values of $\rho$. Since the analysis for these figures is similar to the above analysis of optimal investment strategy of investor A, we omit it here. 


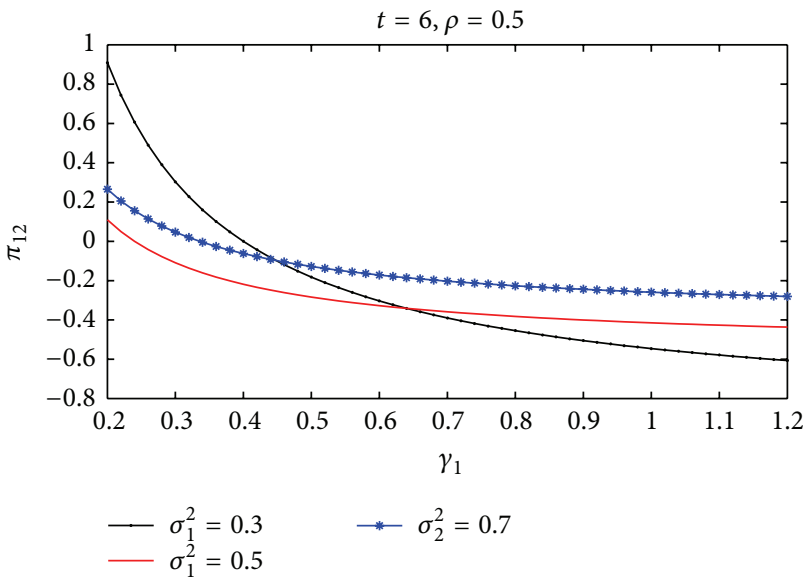

FIGURE 15: $\pi_{1}$ in E2 against $\gamma_{1}$ for $\rho=0.5$ and different $\sigma_{k}^{2}$.

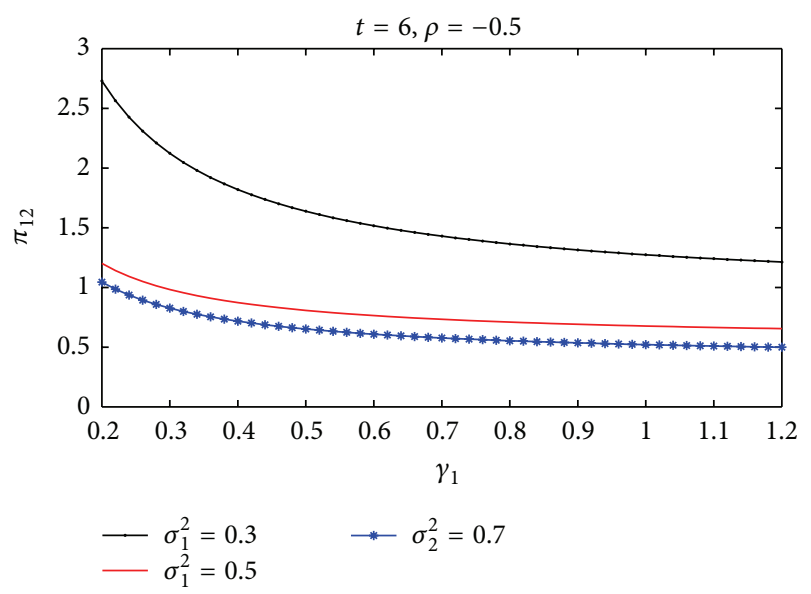

FIGURE 16: $\pi_{1}$ in E2 against $\gamma_{1}$ for $\rho=-0.5$ and different $\sigma_{k}^{2}$.

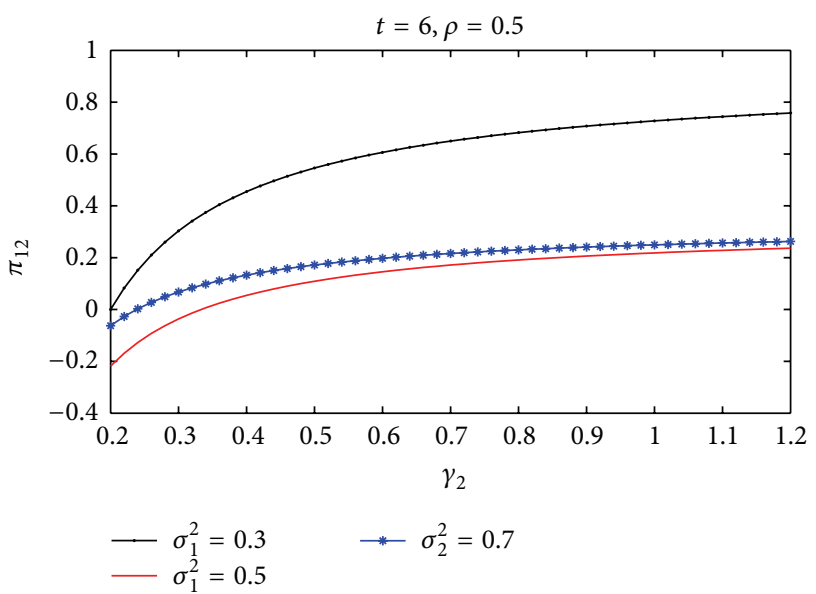

FIGURE 17: $\pi_{1}$ in E2 against $\gamma_{2}$ for $\rho=0.5$ and different $\sigma_{k}^{2}$.

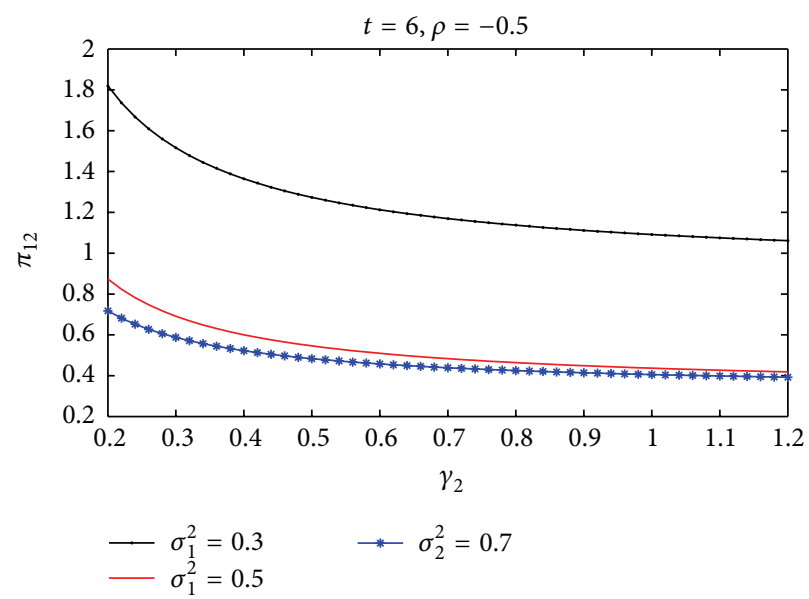

FIGURE 18: $\pi_{1}$ in E2 against $\gamma_{2}$ for $\rho=-0.5$ and different $\sigma_{k}^{2}$.

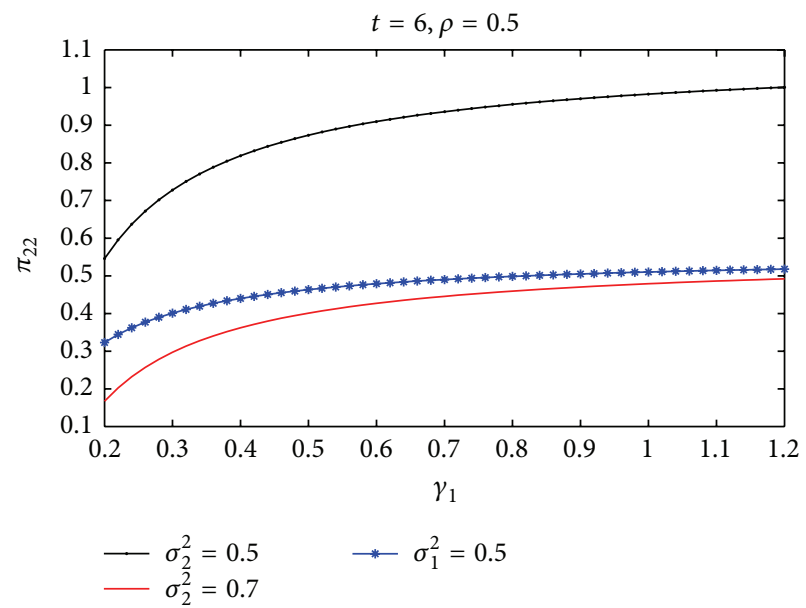

FIGURE 19: $\pi_{2}$ in E2 against $\gamma_{1}$ for $\rho=0.5$ and different $\sigma_{k}^{2}$.

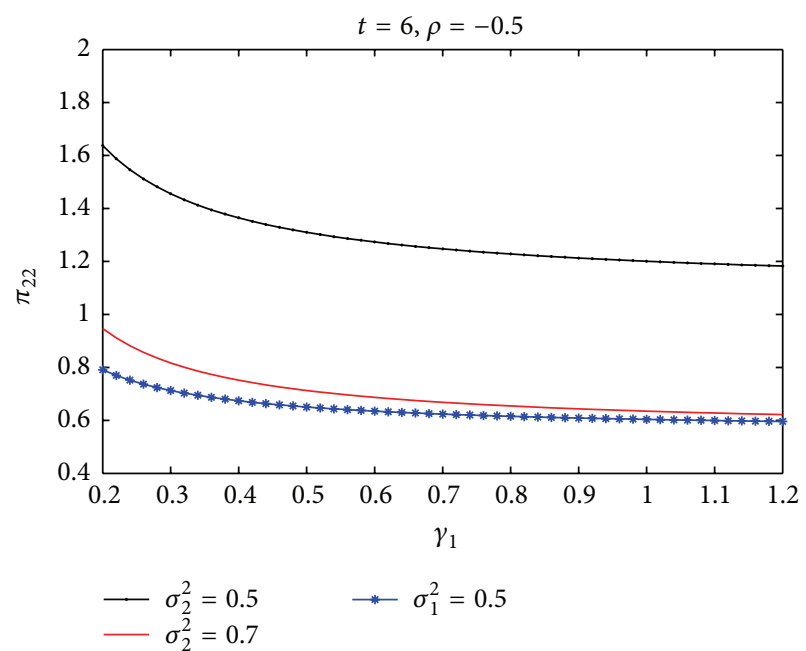

FIgURE 20: $\pi_{2}$ in E2 against $\gamma_{1}$ for $\rho=-0.5$ and different $\sigma_{k}^{2}$. 


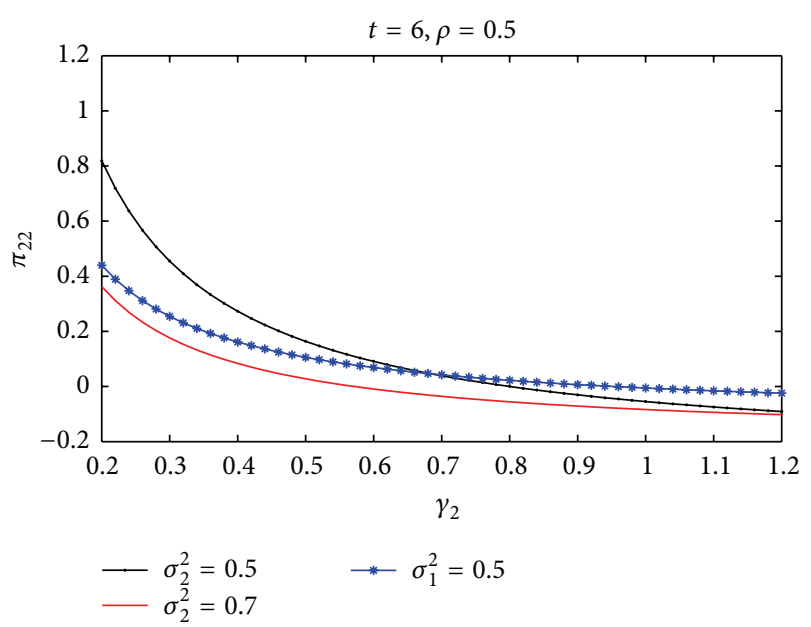

FIGURE 21: $\pi_{2}$ in E2 against $\gamma_{2}$ for $\rho=0.5$ and different $\sigma_{k}^{2}$.

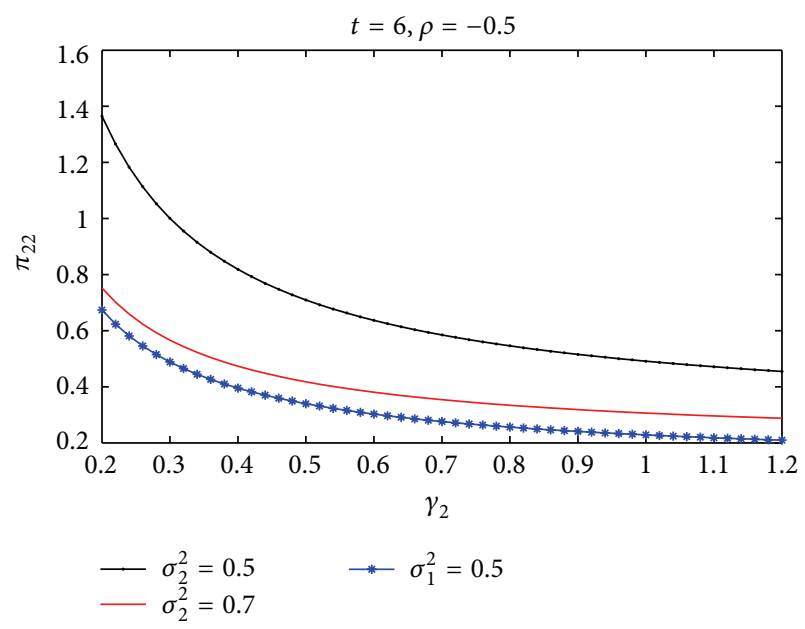

FIGURE 22: $\pi_{2}$ in E2 against $\gamma_{2}$ for $\rho=-0.5$ and different $\sigma_{k}^{2}$.

\section{Conclusion}

In this paper we dealt with a nonzero-sum stochastic differential portfolio game problem between two investors in a continuous-time Markov regime switching model. We formulated the stochastic differential game as two utility maximization problems. We derived a pair of regime switching HJB equations for this differential game problem and then obtained two systems of coupled regime switching HJB equations at different regimes. Explicit solutions to the optimal investment strategies of two investors were also obtained. Furthermore, we derived the Feynman-Kac representations of the value functions of the two utility maximization problems. Numerical results for model parameters and the impact of the regime switching were illustrated and discussed when the Markov chain was assumed to only have two states. It showed that the regime switching in the model parameters had a significant impact on the optimal portfolio strategies.

There are some interesting and potential topics for future research. Firstly, the present paper assumes that the Markov chain can be observed. However, it is interesting to consider a nonzero-sum stochastic differential game under a hidden Markovian regime switching economy. Secondly, we could formulate the nonzero-sum stochastic differential game as a mean-variance portfolio selection problem under a regime switching economy.

A recent related publication by Bensoussan et al. (2014) [42] studied noncooperative nonzero sum games and the problem is formulated as utility maximization of the difference between the terminal wealth.

\section{Conflict of Interests}

The authors declare that there is no conflict of interests regarding the publication of this paper.

\section{Acknowledgments}

The research for this paper was supported by The Key Project of National Natural Science Fund of China (Project no. 71431008), National Natural Science Foundation of China (Project no. 11271375), National Natural Science Innovation Research Group of China (Project no. 71221001), and Hunan Provincial Innovation Foundation For Postgraduate (Project no. CX2014B134).

\section{References}

[1] H. Markowitz, "Portfolio selection," The Journal of Finance, vol. 7, no. 1, pp. 77-91, 1952.

[2] R. C. Merton, "Lifetime porfolio selection under uncertainty: the continuous-time case," The Review of Economics and Statistics, vol. 51, no. 3, pp. 247-257, 1969.

[3] R. C. Merton, "Optimum consumption and portfolio rules in a continuous-time model," Journal of Economic Theory, vol. 3, no. 4, pp. 373-413, 1971.

[4] R. E. Quandt, "The estimation of the parameters of a linear regression system obeying two separate regimes," Journal of the American Statistical Association, vol. 53, no. 284, pp. 873-880, 1958.

[5] J. D. Hamilton, "A new approach to the economic analysis of nonstationary time series and the business cycle," Econometrica, vol. 57, no. 2, pp. 357-384, 1989.

[6] X. Guo, J. Miao, and E. Morellec, "Irreversible investment with regime shifts," Journal of Economic Theory, vol. 122, no. 1, pp. 37-59, 2005.

[7] H. Chen, "Macroeconomic conditions and the puzzles of credit spreads and capital structure," Journal of Finance, vol. 65, no. 6, pp. 2171-2212, 2010.

[8] F. Wen and X. Yang, "Skewness of return distribution and coefficient of risk premium," Journal of Systems Science \& Complexity, vol. 22, no. 3, pp. 360-371, 2009.

[9] F. Wen and Z. Liu, "A copula-based correlation measure and its application in chinese stock market," International Journal of Information Technology and Decision Making, vol. 8, no. 4, pp. 787-801, 2009.

[10] N. Bauerle and U. Rieder, "Portfolio optimization with Markovmodulated stock prices and interest rates," IEEE Transactions on Automatic Control, vol. 49, no. 3, pp. 442-447, 2004. 
[11] R. J. Elliott and T. K. Siu, "On risk minimizing portfolios under a Markovian regime-switching Black-Scholes economy," Annals of Operations Research, vol. 176, no. 1, pp. 271-291, 2010.

[12] R. J. Elliott, T. K. Siu, and A. Badescu, "On mean-variance portfolio selection under a hidden Markovian regime-switching model," Economic Modelling, vol. 27, no. 3, pp. 678-686, 2010.

[13] T. Honda, "Optimal portfolio choice for unobservable and regime-switching mean returns," Journal of Economic Dynamics and Control, vol. 28, no. 1, pp. 45-78, 2003.

[14] K. C. Yiu, J. Liu, T. K. Siu, and W.-K. Ching, "Optimal portfolios with regime switching and value-at-risk constraint," Automatica, vol. 46, no. 6, pp. 979-989, 2010.

[15] X. Zhang and T. K. Siu, "Optimal investment and reinsurance of an insurer with model uncertainty," Insurance: Mathematics \& Economics, vol. 45, no. 1, pp. 81-88, 2009.

[16] X. Y. Zhou and G. Yin, "Markowitz's mean-variance portfolio selection with regime switching: a continuous-time model," SIAM Journal on Control and Optimization, vol. 42, no. 4, pp. 1466-1482, 2003.

[17] R. J. Elliott, "The existence of value in stochastic differential games," SIAM Journal on Control and Optimization, vol. 14, no. 1, pp. 85-94, 1976.

[18] W. H. Fleming and P. E. Souganidis, "On the existence of value functions of two-player, zero-sum stochastic differential games," Indiana University Mathematics Journal, vol. 38, no. 2, pp. 293-314, 1989.

[19] D. W. K. Yeung, "A stochastic differential game of institutional investor speculation," Journal of Optimization Theory and Applications, vol. 102, no. 3, pp. 463-477, 1999.

[20] S. Browne, "Stochastic differential portfolio games," Journal of Applied Probability, vol. 37, no. 1, pp. 126-147, 2000.

[21] F. Wen, Z. He, Z. Dai, and X. Yang, "Characteristics of investors' risk preference for stock markets," Economic Computation and Economic Cybernetics Studies \& Research, vol. 3, no. 48, pp. 235254, 2014.

[22] J. Liu, M. Tao, C. Ma, and F. Wen, "Utility indifference pricing of convertible bonds," International Journal of Information Technology and Decision Making, vol. 13, no. 2, pp. 439-444, 2013.

[23] S. Mataramvura and B. Øksendal, "Risk minimizing portfolios and HJBI equations for stochastic differential games," Stochastics, vol. 80, no. 4, pp. 317-337, 2008.

[24] D. W. Yeung and L. A. Petrosyan, Cooperative Stochastic Differential Games, Springer Series in Operations Research and Financial Engineering, Springer, New York, NY, USA, 2006.

[25] P. Mannucci, "Nonzero-sum stochastic differential games with discontinuous feedback," SIAM Journal on Control and Optimization, vol. 43, no. 4, pp. 1222-1233, 2004.

[26] R. J. Elliott and T. K. Siu, "A Markovian regime-switching stochastic differential game for portfolio risk minimization," in Proceedings of the American Control Conference (ACC '08), pp. 1017-1022, Seattle, Wash, USA, June 2008.

[27] T. K. Siu, "A game theoretic approach to option valuation under Markovian regime-switching models," Insurance: Mathematics and Economics, vol. 42, no. 3, pp. 1146-1158, 2008.

[28] C. K. Leong and W. Huang, "A stochastic differential game of capitalism," Journal of Mathematical Economics, vol. 46, no. 4, pp. 552-561, 2010.

[29] X. Lin, "Nonzero-sum stochastic differential portfolio games," Working Paper, Zhenjiang Gongshang University, 2013.
[30] R. J. Elliott and T. K. Siu, "A stochastic differential game for optimal investment of an insurer with regime switching," Quantitative Finance, vol. 11, no. 3, pp. 365-380, 2011.

[31] J. Z. Liu and K. F. C. Yiu, "Optimal stochastic differential games with VaR constraints," Discrete and Continuous Dynamical Systems Series B, vol. 18, no. 7, pp. 1889-1907, 2013.

[32] H. Yang and L. Zhang, "Optimal investment for insurer with jump-diffusion risk process," Insurance: Mathematics \& Economics, vol. 37, no. 3, pp. 615-634, 2005.

[33] R. J. Elliott, L. Aggoun, and J. Moore, Hidden Markov Models :Estimation and Control, Springer, New York, NY, USA, 1995.

[34] M. I. Kamien and N. L. Schwartz, Dynamic Optimization: The Calculus of Variations and Optimal Control in Economics and Management, North Holland, Amsterdam, The Netherlands, 2nd edition, 1991.

[35] F. Wen, Z. He, and X. Chen, "Investors' risk preference characteristics and conditional skewness," Mathematical Problems in Engineering, vol. 2014, Article ID 814965, 14 pages, 2014.

[36] F. Wen, Z. He, X. Gong, and A. Liu, "Investors' risk preference characteristics based on different reference point," Discrete Dynamics in Nature and Society, vol. 2014, Article ID 158386, 9 pages, 2014.

[37] C. Huang, H. Kuang, X. Chen, and F. Wen, "An LMI approach for dynamics of switched cellular neural networks with mixed delays," Abstract and Applied Analysis, vol. 2013, Article ID 870486, 8 pages, 2013.

[38] G. L. Esparza, M. G. Torres, and S. L. Torres, "A brief introduction to differential games," International Journal of Physical and Mathematical Sciences, vol. 4, no. 1, 2013.

[39] W. H. Fleming and H. M. Soner, Controlled Markov Processes and Viscosity Solutions, Springer, New York, NY, USA, 2006.

[40] R. Bronson, Matrix Methods: An Introduction, Academic Press, London, UK, 1991.

[41] X. Zhang and T. K. Siu, "On optimal proportional reinsurance and investment in a Markovian regime-switching economy," Acta Mathematica Sinica, vol. 28, no. 1, pp. 67-82, 2012.

[42] A. Bensoussan, C. C. Siu, S. C. Yam, and H. Yang, "A class of non-zero-sum stochastic differential investment and reinsurance games," Automatica, vol. 50, no. 8, pp. 2025-2037, 2014. 


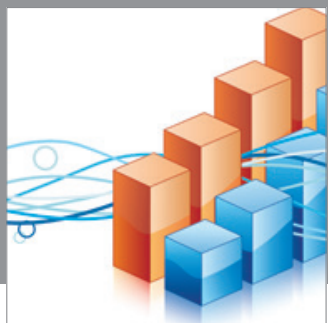

Advances in

Operations Research

mansans

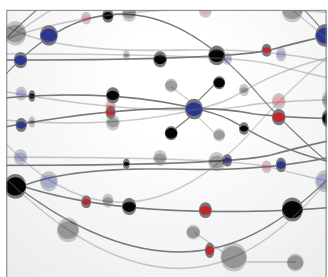

The Scientific World Journal
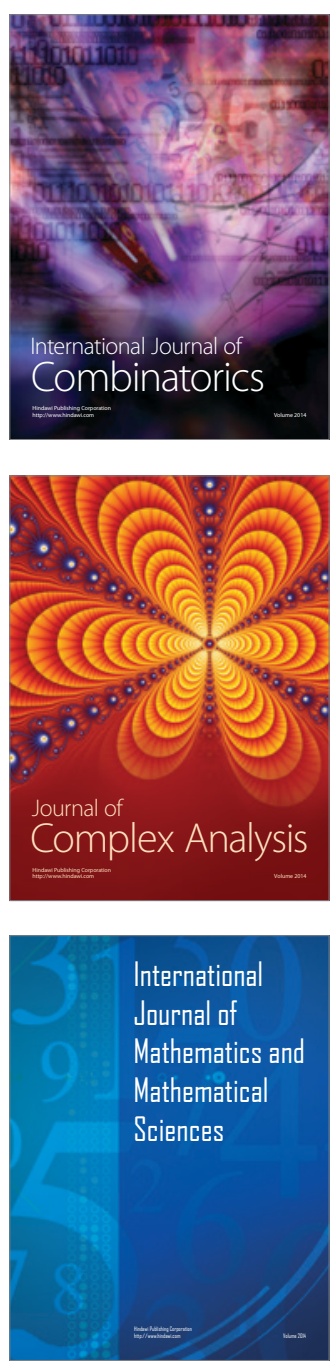
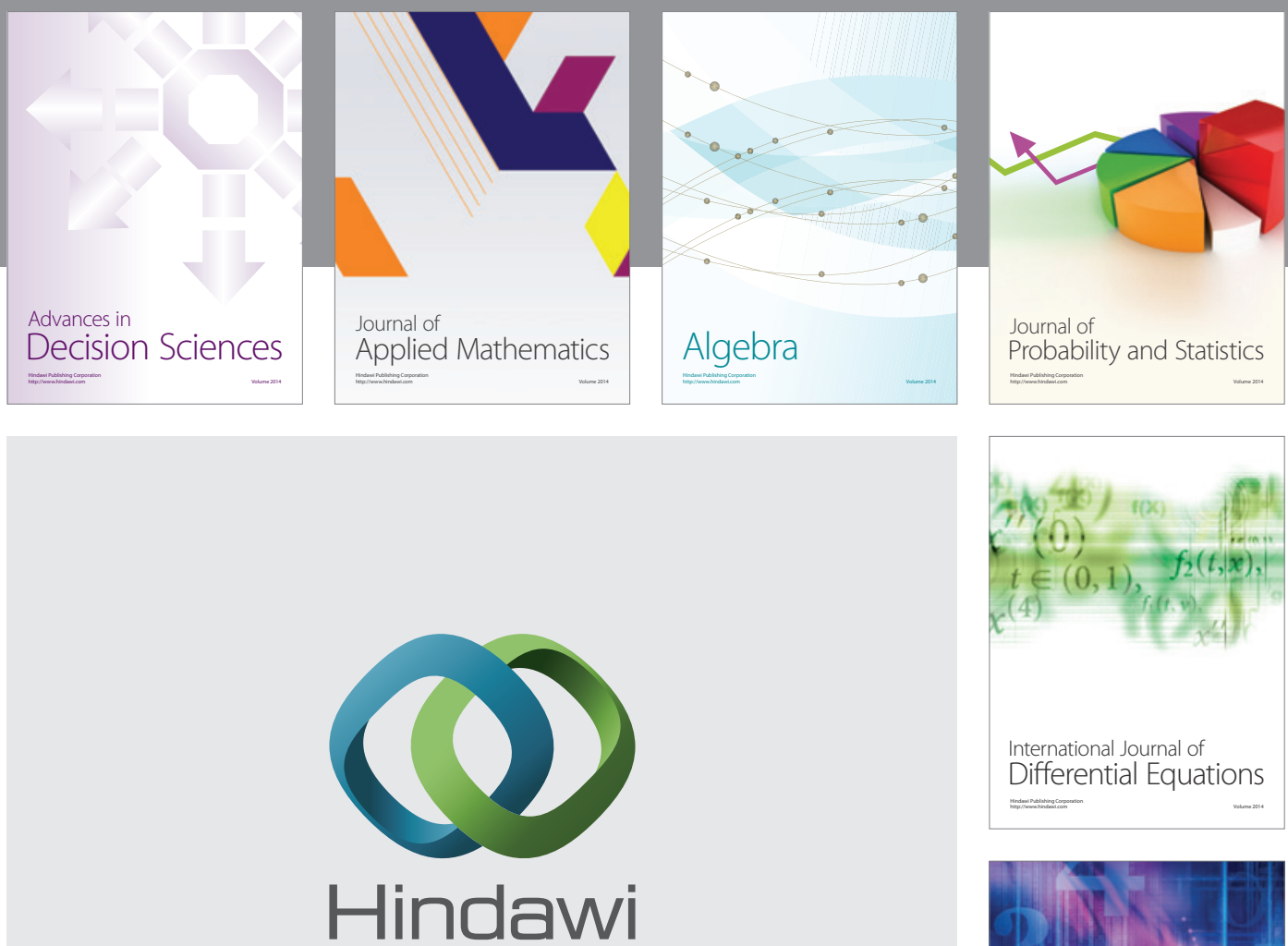

Submit your manuscripts at http://www.hindawi.com
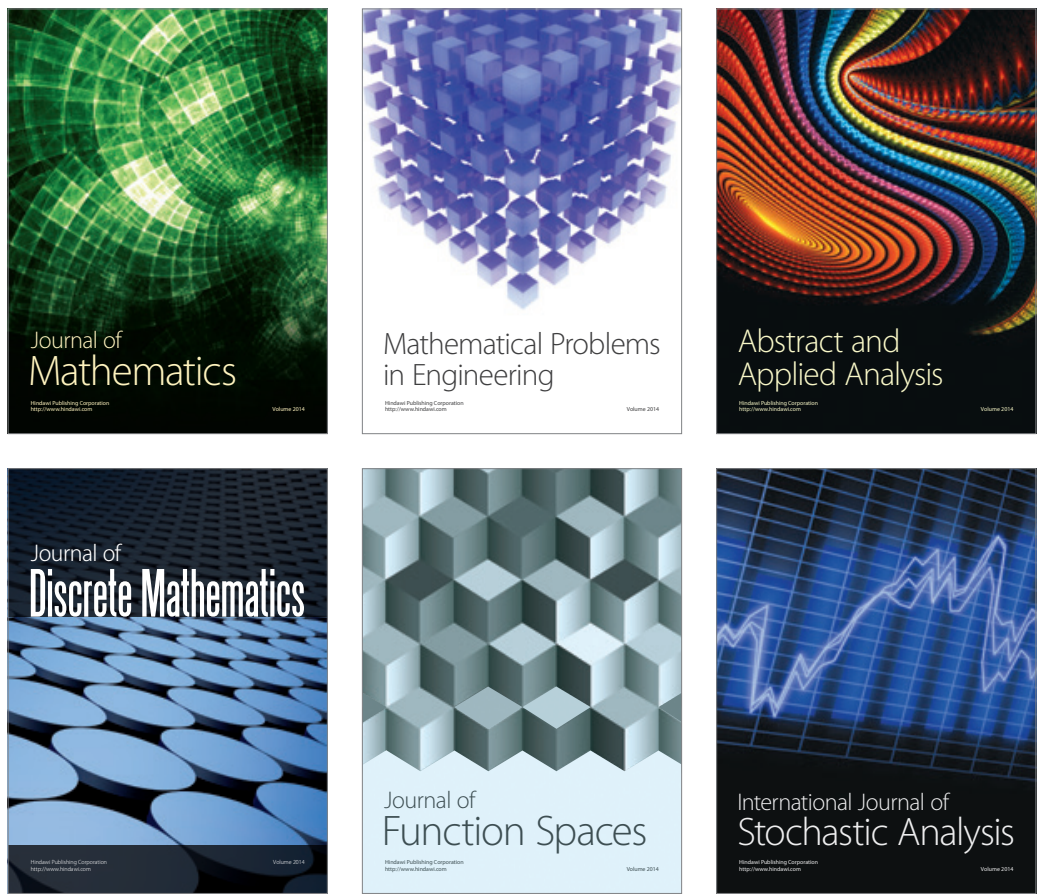

Journal of

Function Spaces

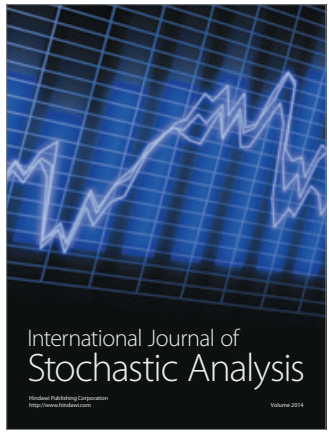

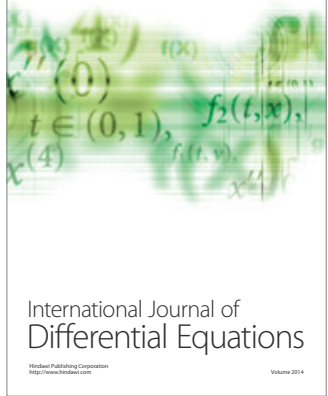
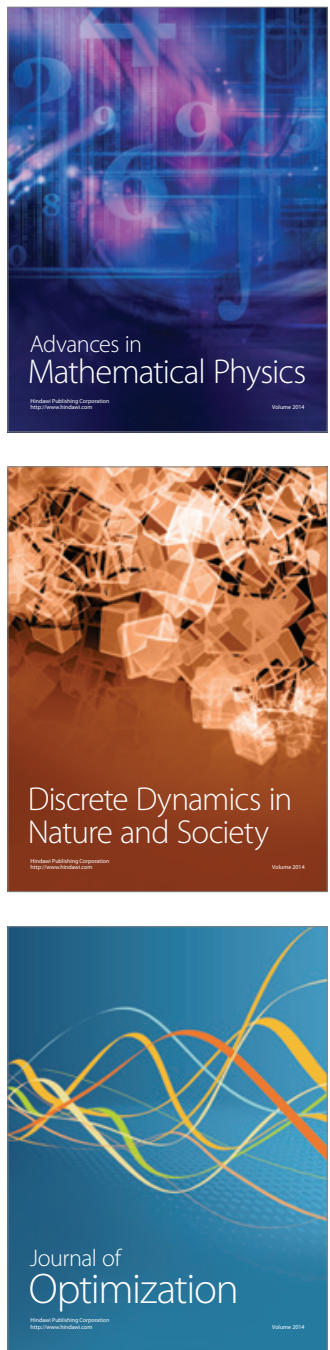